PRAXIS

ecucativa

Universidad Nacional de La Pampa

Facultad de Ciencias Humanas

Instituto de Ciencias de la Educación

para la investigación interdisciplinaria

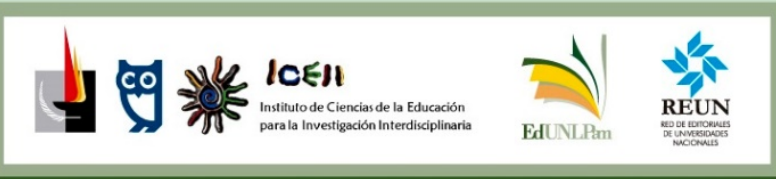

ISSN 2313-934X

SANTA ROSA, LA PAMPA, ARGENTINA

Correo electrónico: iceii@humanas.unlpam.edu.ar

Disponible en https://cerac.unlpam.edu.ar/index.php/praxis

Estudio bibliométrico sobre la Pedagogía de la Alternancia en artículos científicos (1981-2019). Artículo de María Cristina Piumbato Innocentini Hayashi; Alexandre Masson Maroldi; Taísa Grasiela Gomes Liduenha Gonçalves; Carlos Roberto Massao Hayashi. Praxis educativa, Vol. 26, No 1 enero - abril 2022. E - ISSN 2313-934X. pp. 1-28. https://dx.doi.org/10.19137/praxiseducativa-2022-260107

Esta obra se publica bajo Licencia Creative Commons 4.0 Internacional CC BY- NC-SA Atribución, No Comercial, Compartir igual

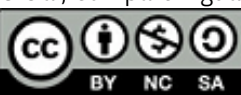

\title{
Estudio bibliométrico sobre la Pedagogía de la Alternancia en artículos científicos (1981-2019)
}

Bibliometric study on the Pedagogy of Alternance in scientific articles (1981-2019)

Estudo bibliométrico sobre a Pedagogia da Alternância em artigos científicos (1981-2019)

\section{María Cristina Piumbato Innocentini Hayashi}

Universidade Federal de São Carlos, SP, Brasil

dmch@ufscar.br

ORCID 0000-0003-1250-3767

\section{Alexandre Masson Maroldi}

Universidade Federal de Rondônia, RO, Brasil

alexandre@unir.br

ORCID 0000-0002-6592-7750

\section{Taísa Grasiela Gomes Liduenha Gonçalves}

Universidade Federal de Minas Gerais, MG, Brasil

taisaliduenha@gmail.com

ORCID 0000-0001-5589-584X

\section{Carlos Roberto Massao Hayashi}

Universidade Federal de São Carlos, SP, Brasil

massao@ufscar.br

ORCID 0000-0003-1481-5545

Recibido: 2021-04-15 | Revisado: 2021-08-23 | Aceptado: 2021-09-19 


\section{Resumen}

La Pedagogía de la Alternancia (AP) es una propuesta formativa y educativa del ser humano que organiza el proceso de enseñanza-aprendizaje, alternando espacios entre propiedad familiar y escuela. Este estudio tiene como objetivo comprender cómo se configura el estado actual del conocimiento sobre esta temática, a través de la metodología de análisis bibliométrico y de contenido. Las fuentes de datos fueron artículos indexados en: Web of Science, Scopus, Redalyc, DOAJ, SciELO, Educ@, EDUBASE y plataforma digital de Periódicos Capes. Los artículos seleccionados ( $n=251$ ) fueron publicados entre 1981 y 2019, y analizados según las categorías de: temporalidad, autores y autoría, perfil de revistas, tipología de artículos, locus y objetos de estudio de la investigación empírica, alcance de los ensayos teóricos y análisis crítico del metanálisis. Los resultados permitieron identificar las perspectivas longitudinales y estructurales de la producción científica en Pedagogía de la Alternancia y sus vínculos con diversos contextos disciplinarios e interdisciplinarios.

Palabras-clave: Pedagogía de la Alternancia; estado del conocimiento; producción científica; análisis bibliométrico y de contenido

\section{Abstract}

Pedagogy of Alternation (PA) is a formative and educational proposal of the human being that organizes the teaching-learning process, alternating spaces between family property and school. This study aims to understand how the current state of knowledge on this theme is configured, through the methodology of bibliometric and content analysis. The data source was articles indexed on the Web of Science, Scopus, Redalyc, DOA), SciELO, Educ @, EDUBASE, Portal de Periódicos Capes. The selected articles ( $n=251$ ) were published between 1981 and 2019, and analyzed according to the categories of: temporality, authors and authorship, profile of journals, typology of articles, locus and objects of study of empirical research, scope of theoretical essays and critical analysis of meta-analyzes. The results made it possible to identify the longitudinal and structural perspectives of scientific production in Pedagogy of Alternation and their links with various disciplinary and interdisciplinary contexts.

Keywords: Pedagogy of Alternation; state of knowledge; scientific production; bibliometric; content analysis

\section{Resumo}

A Pedagogia da Alternância (PA) é uma proposta formativa e educacional do ser humano que organiza o processo de ensino-aprendizagem alternando espaços da propriedade familiar e da escola. Esse estudo visa compreender como se configura o estado do conhecimento sobre essa temática passados cinquenta anos da introdução dessa proposta educativa no Brasil. As abordagens metodológicas adotadas foram a análise bibliométrica e de conteúdo. As fontes de dados foram artigos indexados na Web of Science, Scopus, Redalyc, DOA), SciELO, Educ@, EDUBASE, Portal de Periódicos Capes. Os artigos selecionados ( $n=251$ ) foram publicados entre 1981 e 2019 e analisados segundo as categorias de: temporalidade, autores e autorias, perfil dos periódicos, tipologia dos artigos, lócus e objetos de estudo das pesquisas empíricas, escopo dos ensaios teóricos e análise crítica das metanálise. Os resultados permitiram identificar as perspectivas longitudinais e estruturais da produção científica em Pedagogia da Alternância e suas ligações com vários contextos disciplinares e interdisciplinares.

Palavras-chave: Pedagogia da alternância; estado do conhecimento; produção científica; análise bibliométrica; análise de conteúdo 


\section{Introducción}

Anclada en un compromiso político de rechazo a la discriminación del hombre y de la cultura del campo, por medio de un proceso educativo que integra a la familia y a la comunidad, y basado en una relación dialéctica entre teoría y práctica, la propuesta formativa de personas jóvenes y adultas de la Pedagogía de la Alternancia visa a la formación integral y sociocomunitaria del ser humano, en oposición a los modelos tradicionales de educación marcados por el individualismo (Nosella, 2007). En otras palabras, en la Pedagogía de la Alternancia, la formación integral, el desarrollo del entorno, la alternancia y la asociación constituyen los cuatro momentos significativos de este proceso de enseñanza y aprendizaje (Gimonet, 2007, García-Marirrodriga y Calvó, 2010). En ese contexto, como refiere Ribeiro (2010), el elemento fundamental es el "trabajo como principio educativo de una formación humana integral, que articula dialécticamente el trabajo productivo a la enseñanza formal" (p. 293) (traducción libre del original en portugués).

Las raíces históricas de la Pedagogía de la Alternancia (PA) se pueden encontrar en experiencias educativas de origen francés (Chartier,1985) desarrolladas en el contexto de las Maisons Familiares Rurales, y que se han esparcido por países de Europa, África, Asia y América (Miano y Corro, 2018). En Brasil, la Pedagogía de la Alternancia se introdujo en 1969, a través de la acción pastoral de los jesuitas y liderada por el Movimiento de Educación Promocional del Espírito Santo (MEPES), con el apoyo de líderes comunitarios de la región sur de ese Estado, según informan Nosella $(1970,1978,2007,2012)$ y Pessotti $(1978,1981)$. Las experiencias y prácticas educativas de la Pedagogía de la Alternancia se materializaron en las Casas Familiares Rurales (CFRs), Centros Familiares de Formación por Alternancia (CEFFAs) y Escuelas Familia Agrícola (EFAs), que suman 270 unidades existentes en todo el país y distribuidos en 21 estados y 800 municipios (Nosella, 2012). Desde entonces, esta experiencia educativa se ha extendido por todo el país, tanto en el contexto de los movimientos sociales en el campo, en una forma de resistencia al proceso de expansión del capitalismo, como en la formación educativa crítica y humanística de jóvenes y adultos que valoran sus raíces culturales, al mismo tiempo que lleva a estos estudiantes a superar la visión del campo únicamente como un espacio de producción.

En Brasil, los dos primeros trabajos académicos de posgrado sobre la PA fueron presentados en alrededor de 1970, bajo orientación de Dermeval Saviani (Nosella, 1977) y Omar Fávero (Pessotti, 1978). Cabe señalar que Paolo Nosella, investigador italiano radicado en el país, tuvo una participación activa en la implantación de la PA en Brasil, y sus trabajos han sido tomados como referencia teórica fundamental para la investigación sobre este tema.

Así, este artículo se guía por la búsqueda de respuestas a la siguiente pregunta de investigación: ¿cómo se configura la producción científica sobre Pedagogía de la Alternancia? El objetivo de la investigación es realizar un estado de conocimiento de la producción científica sobre este tema. La metodología adoptada se describe en el siguiente apartado, seguido de la presentación y discusión de los resultados obtenidos. 


\section{Metodología}

La investigación realizada es de tipo bibliográfico, exploratorio y descriptivo, y puede caracterizarse como un estado del arte también denominado estado de conocimiento o metanálisis-, ya que contribuye a sintetizar y analizar el conocimiento producido sobre un tema determinado, disciplina o área científica (Pizzani et al., 2013; Hayashi y Gonçalves, 2018). La metodología empleó análisis bibliométrico y de contenido combinados, que consisten en un conjunto de métodos cuantitativos y cualitativos para desarrollar indicadores de producción científica y realizar descripciones objetivas de los mensajes expresados en los textos, permitiendo comprender la estructura social e intelectual de un campo científico y la inferencia de conocimientos extraídos de los documentos analizados (Silva et al., 2011; Bardin, 2011). Esa metodología ya ha sido utilizada por los autores en otros estudios sobre la educación del campo (Gonçalves y Hayashi, 2014, 2016; Hayashi y Gonçalves, 2016) y la educación indígena (Maroldi et al., 2018; Maroldi et al., 2018), mostrándose adecuada para su replicación en el presente estudio. Además, al asociar métodos estadísticos, sociológicos y lingüísticos, esta metodología multidisciplinaria fortalece los análisis y amplía las posibilidades de discusión de los hallazgos.

Los artículos científicos fueron elegidos como objeto de estudio de la producción científica sobre PA por su papel central en el contexto de la producción y difusión del conocimiento científico a través de canales formales de comunicación, aunque se reconoce la importancia de otros tipos de producción científica como libros, capítulos de libros y trabajos presentados en eventos. A esto se suma, como se verá más adelante en la descripción y discusión de los resultados de la investigación aquí reportada, el hecho de que la mayoría de los balances de producción científica sobre Pedagogía de la Alternancia tomó tesis de magíster y de doctorado como objeto de estudio, sugiriendo que esta tipología documental ha sido ampliamente explorada y reforzando la motivación para explorar otras fuentes bibliográficas, como se llevó a cabo en el presente estudio.

Con la intención de recuperar la literatura científica en armonía con el tema de investigación, se utilizaron como fuentes de datos ocho plataformas de información científica brasileña, latinoamericana y mundial, seleccionadas por su amplia visibilidad y cobertura geográfica, que incluye literatura científica de países de América Latina y el Caribe, España, Portugal, Alemania, Dinamarca, Estados Unidos y Polonia, y también porque abarcan varias áreas del conocimiento, principalmente Humanidades y Ciencias Sociales, además de ofrecer acceso abierto a los documentos. La recopilación de datos tuvo lugar en mayo de 2019, utilizando las expresiones de búsqueda "pedagogia da alternância", "pedagogía de la alternancia", "pedagogy of alternancy" y "pédagogie de l'alternance", lo que resultó en la recuperación inicial de 304 artículos así distribuidos: Plataforma de Periódicos Capes (Coordinación de Perfeccionamiento de Personal de Nivel Superior del Ministerio de Educación brasileño) $(n=84)$, Directory of Open Access Journals $(D O A J)(n=75)$, Red de revistas científicas Redalyc $(n=74)$, Web of Science (WoS) $(n=37)$, EDUBASE $(n=18)$, Scopus $(n=8)$, Educ@ $(n=6)$ y biblioteca electrónica SciELO $(n=2)$. 
Estudio bibliométrico sobre la Pedagogía de la Alternancia en artículos científicos (1981-2019). I

María Cristina Piumbato Innocentini Hayashi; Alexandre Masson Maroldi; Taísa Grasiela Gomes Liduenha Gonçalves; Carlos Roberto

Massao Hayashi.

Como criterio de inclusión, se estableció que no se iba a poner un marco temporal en cuanto a los resultados obtenidos para mapear toda la literatura científica existente sobre este tema en las bases de datos consultadas y que los artículos deberían tener disponible el texto completo. Los criterios de exclusión fueron los artículos presentes en más de una base de datos y aquellos que no cumplían con el alcance de la investigación caracterizados por citar la Pedagogía de la Alternancia solo en el resumen o en una oración del texto, abordándola solamente como un trasfondo para introducir un tema y objeto de estudio sin adherirse al tema investigado. Luego de aplicar estos criterios, se excluyeron 53 artículos, resultando un corpus final de 251 artículos que fueron publicados entre 1981 y 2019.

Los procedimientos metodológicos se realizaron en las siguientes fases: 1) recolección y registro de datos en el portafolio de investigación elaborado con la ayuda del software Excel; 2) modelado de datos para eliminar inconsistencias en la nomenclatura de títulos de revistas y nombres de autores; 3 ) lectura completa de los artículos para establecer las categorías de análisis; 4) cruce, síntesis y descripción de los datos; 5) análisis e interpretación de resultados.

Las categorías de análisis se basaron en parámetros bibliométricos y de contenido de los artículos conteniendo las siguientes variables: a) perfil de los artículos: evolución temporal y autores (género y nacionalidad), autoría (individual y en coautoría) y publicaciones en revistas (títulos y países); b) tipología de artículos (estudios de caso, ensayos teóricos y metanálisis); c) locus y objetos de estudio de las investigaciones empíricas; d) alcance de los ensayos teóricos; e) análisis crítico de metanálisis (período abarcado, fuente de datos, temas y categorías de análisis).

Las divergencias encontradas en esta categorización fueron discutidas por los/as autores/as y resueltas por consenso. A continuación, se presentan los resultados obtenidos en dos claves de análisis: la primera ofrece un panorama bibliométrico de la literatura científica sobre Pedagogía de la Alternancia, destacando los principales indicadores de este tipo de publicación; el segundo muestra el contenido de los artículos, caracterizando los principales aspectos teóricos y metodológicos de esta producción científica.

\section{Resultados}

La organización de los resultados permitió trazar la evolución temporal (Figura 1) de los artículos ( $n=251$ ) sobre Pedagogía de la Alternancia publicados entre 1981 y 2019. 


\section{Figura 1}

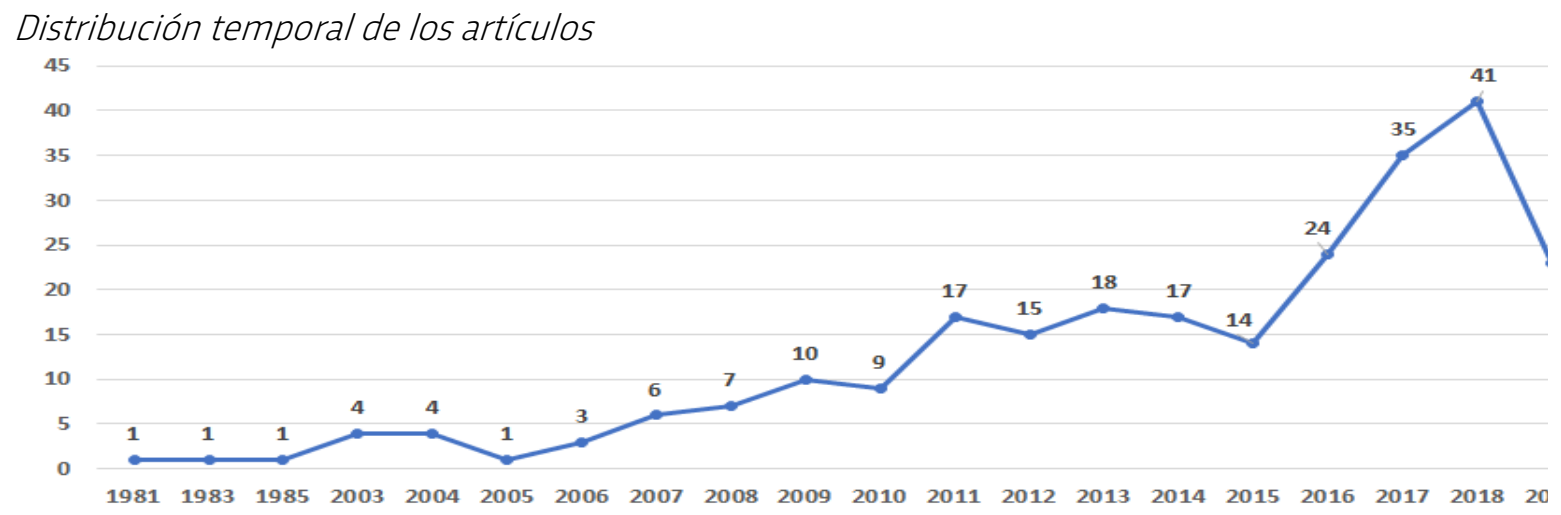

No se ha recuperado producción científica publicada alrededor de 1970, si bien, en esa década, Nosella había publicado dos artículos $(1970,1978)$. Aunque hayan aparecido en las búsquedas realizadas, se justifica su no inclusión por el hecho de que los textos completos no están disponibles en línea en las bases de datos consultadas. ${ }^{1}$

Asimismo, se observa que la producción científica en la década de 1980 es incipiente y está representada por solo tres artículos (Pessotti, 1981; Argumedo, 1983; Chartier, 1985), resultado relacionado con los hallazgos de Teixeira et al. (2008), que identificaron, en ese período, la producción de solo una tesis de magíster sobre la Pedagogía de la Alternancia (Gioanordoli, 1980).

La Figura 1 todavía revela que hay un vacío en la publicación de artículos en la década de los noventa, volviendo con publicaciones desde 2003 y en los próximos cuatro años hasta 2006, cuando hay un crecimiento anual significativo. En los años que siguieron hasta 2009, hubo un incremento en el promedio anual $(n=7,6)$, aunque todavía bajo si se compara con el crecimiento significativo de la producción científica en la década de 2010, que presenta un promedio anual alto $(n=21,3)$, con la culminación de artículos en el año $2018(n=45)$. Cabe señalar, también, que el nivel del año $2019(n=23)$ es relativamente alto, considerando que la recolección de datos ocurrió en mayo de 2019.

El análisis de las autorías de los artículos reveló que las autorías individuales fueron minoría $(n=77)$ comparadas a las coautorías $(n=174)$ y, en estas, prevalecieron las coautorías dobles $(n=116)$ y triples $(n=42)$. Aunque más raro en relación con los demás, se identificaron coautorías de cuatro $(n=9)$, cinco $(n=5)$ y ocho $(n=1)$ autores. Estos resultados confirman que, en el área de las Ciencias Humanas, ha cambiado el estándar de comunicación científica más centrado en la autoría individual, como lo evidencian los estudios en el área de Educación, realizados por Lopes y Costa (2012), y en el campo de la Sociología política, de Soares et al. (2010), quienes mostraron un incremento de la coautoría en estos campos del conocimiento.

Al considerar el género de los autores $(n=408)$, la investigación reveló (Figura 2$)$ que las mujeres $(n=241)$ son mayoría, con el $59 \%$ de la totalidad, y los hombres representaron el $41 \%$ ( $n$ $=167)$. 


\section{Figura 2}

Género de los/as autores/as brasileños/as y extranjeros/as de acuerdo con los países

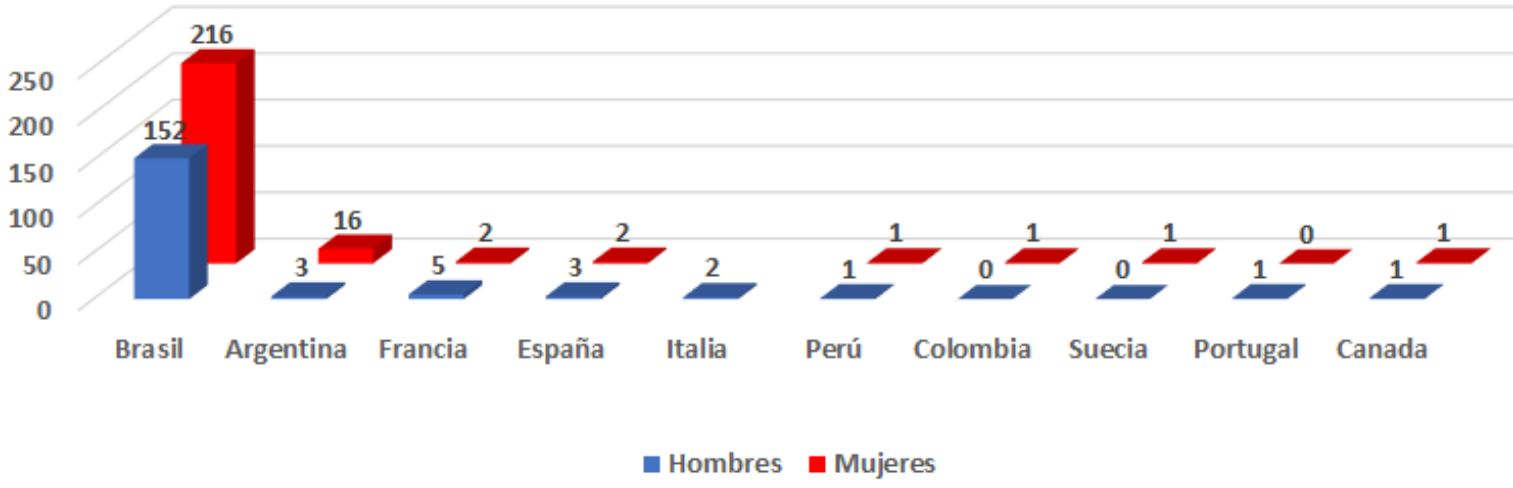

Este perfil mayoritariamente femenino en las autorías presentes en la producción científica sobre PA reproduce, en alguna medida, la feminización del campo científico de la Educación. En esta área del conocimiento, así como en otros campos de las Humanidades, está presente el fenómeno social de la segregación horizontal en la ciencia (Schiebinger, 1999), es decir, cuando las normas prescriptivas y los estereotipos sociales culturalmente considerados femeninos o masculinos influyen en las elecciones profesionales de los individuos en determinadas áreas y especialidades. En cuanto a la nacionalidad de los autores $(n=408)$, los resultados indicaron que la mayoría $(n=368)$ está compuesta de investigadores brasileños y, los demás, el 9,8\% $(n=40)$, son de otros países. El cruce de los datos sobre género y nacionalidad también reveló que las mujeres prevalecen con relación a los hombres.

En cuanto a los países de las revistas $(n=157)$ que publicaron los artículos ( $n=251)$, la distribución se puede ver en la Figura 3.

\section{Figura 3}

Distribución de los artículos de acuerdo con los países de las revistas

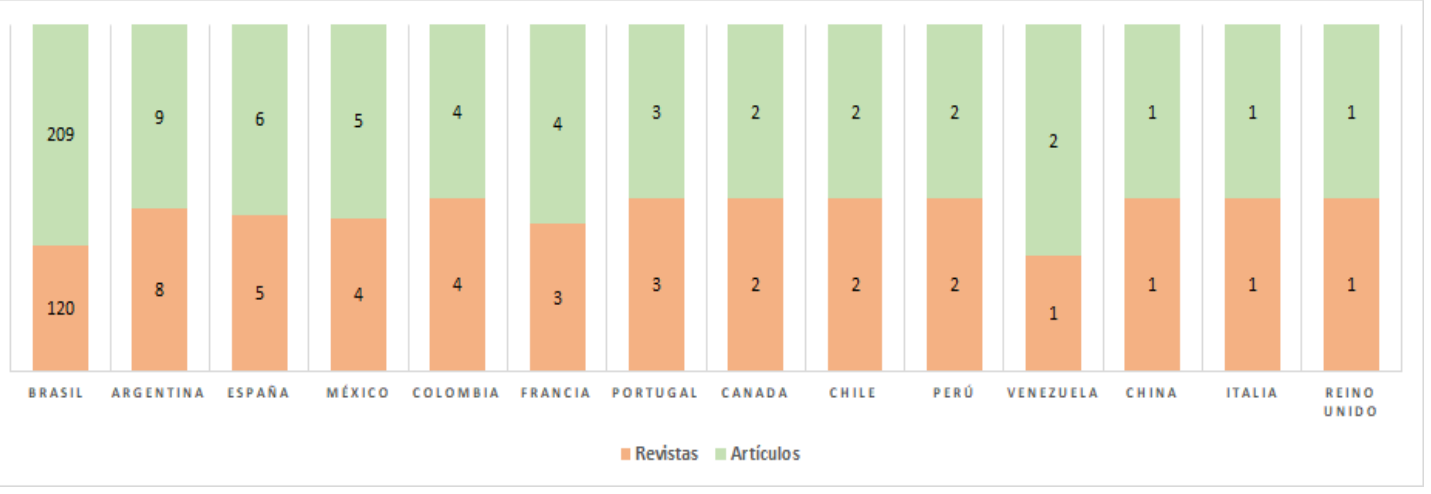


Los datos de la Figura 3 revelan que el 76,4\% $(n=120)$ de la totalidad de títulos está constituido por revistas brasileñas, que han divulgado el $83,3 \%(n=209)$ de los artículos, mientras que el $23,6 \%(n=37)$ es de otros países $(n=13)$, con el $16,7 \%(n=42)$, demostrando que la temática de la PA tuvo buena receptividad en esas revistas.

En América del Sur, entre los demás países $(n=5)$ representados, el liderazgo es de Argentina, con la mayoría de las producciones $(n=8)$ y artículos $(n=9)$, seguida de Colombia $(n=$ $4 ; n=4)$, Chile y Perú, con los mismos puntajes $(n=2 ; n=2)$, y Venezuela $(n=1 ; n=2)$. En Europa, España lidera en número de revistas y artículos $(n=5 ; n=6)$, seguida por Francia $(n=$ $3 ; n=4)$ y Portugal $(n=3 ; n=3)$, mientras que Italia y Reino Unido $(n=1$ y $n=1)$ alcanzan los mismos índices. En América del Norte, el liderazgo es de México $(n=4$ y $n=5)$, seguido por Canadá ( $n=2$ y $n=2)$. Asia está representada solo por China, que ocupa la última posición ( $n=1$ y $\mathrm{n}=1$ ) entre los títulos de revistas y artículos publicados.

La Tabla 1 presenta la distribución de los artículos de acuerdo con los títulos de las revistas brasileñas y del exterior.

\section{Tabla 1}

Distribución de los artículos de acuerdo con los títulos de las revistas

\begin{tabular}{|c|c|}
\hline Títulos & Artículos \\
\hline Revista Brasileira de Educação do Campo & 22 \\
\hline Educere et Educare; Revista Histedbr On-line (6 artículos cada) & 12 \\
\hline Revista NERA; Synergismus Scientifica (5 artículos cada) & 10 \\
\hline Reflexão \& Ação; Revista da FAEEBA; Revista Pedagógica (Unochapecó) (4 artículos cada) & 12 \\
\hline $\begin{array}{l}\text { Cadernos CIMEAC; EccoS Revista Científica; Educação \& Realidade; Educação \& Sociedade; } \\
\text { Educação e Pesquisa; Educação em Perspectiva; Itinerarius Reflexionis; Revista Brasileira de } \\
\text { Educação; Revista de Ciências Humanas (URI); Revista Pegada Eletrônica; Revista Práxis } \\
\text { Educacional (3 artículos cada) }\end{array}$ & 33 \\
\hline $\begin{array}{l}\text { Atos de Pesquisa em Educação; Cadernos de Pesquisa Pensamento Educacional; Cadernos } \\
\text { EBAPE.BR; Educação em Revista (Marília); Em Aberto (INEP); Enseñanza de las Ciencias (España); } \\
\text { Entrelaçando: Revista Eletrônica de Culturas e Educação; Espacios (Venezuela); Extensão Rural } \\
\text { (Santa Maria); GEOSABERES; Holos; Inter-Ação; Linhas Críticas; Olhar de Professor; Práxis } \\
\text { Educativa (UEPG); Redes (UCS); Revista de Educação Popular; Revista de Educação, Ciência e } \\
\text { Cultura; Revista Diálogo Educacional; Revista Eventos Pedagógicos; Revista Latinoamericana de } \\
\text { Estudios Educativos (México); Revue Française de Pédagogie (Francia); Tempos e Espaços em } \\
\text { Educação; E+E: Estudios de Extensión en Humanidades (Argentina) (2 artículos cada) }\end{array}$ & 48 \\
\hline 114 revistas con solo un artículo cada & 114 \\
\hline Totalidad & 251 \\
\hline
\end{tabular}

Entre las revistas $(n=157)$, se ha verificado que un pequeño contingente, compuesto por el $27,4 \%(n=43)$ de la totalidad, fue responsable de la publicación de la mayoría de los artículos, representando el $54,6 \%(n=137)$. En cambio, la mayoría de las revistas, representadas por el 
$72,5 \%(n=114)$ de los títulos, publicaron solo un artículo cada una, es decir, el 45,4\% ( $n=114)$ del número total de artículos $(n=251)$. También se observó que el $23,5 \%(n=37)$ de las revistas publicó entre seis y dos artículos, concentrando el $45,8 \%(n=115)$ de los artículos. Esta puntuación se acerca mucho a la obtenida por las revistas $(n=114)$ que publicaron solo un artículo cada una.

Entre las revistas que integran el núcleo principal, se destaca la Revista Brasileira de Educação do Campo, responsable de la publicación del 8,8\% $(n=22)$ de los artículos. Aunque no está dedicado exclusivamente a la Pedagogía de la Alternancia, este tema forma parte del ámbito de interés de la revista. Además, como demuestra Bicalho (2013), existe una estrecha articulación entre Educación del Campo, Pedagogía de la Alternancia y movimientos sociales.

Ocupan la segunda posición en la Tabla 1 los artículos publicados en la revista Educere et Educare $(\mathrm{n}=6)$-que compusieron los dossiers Acción de aprendizaje y enseñanza y Educación del campo- y en la Revista Histedbr Online $(\mathrm{n}=6)$, cuyo alcance abarca la educación como fenómeno social en su conexión con la reflexión histórica. El tema de interés de las otras revistas que publicaron entre cinco y dos artículos abarca, principalmente, el campo de la Educación, con temáticas orientadas a discutir problemas educativos contemporáneos, así como revistas multidisciplinarias, que difunden el conocimiento científico de diferentes áreas del conocimiento. La distribución de los títulos de las revistas $(n=37)$ del exterior reveló el predominio $(n=17)$ de países $(n=5)$ de América Latina, seguidos de las revistas $(n=13)$ de países de Europa $(n=5)$. Las revistas $(n=6)$ de países $(n=2)$ de América del Norte tuvieron la incidencia más baja. Asia estuvo representada por una sola revista.

La Tabla 2 presenta la distribución de los artículos publicados en las revistas de fuera de Brasil.

\section{Tabla 2}

Distribución de los artículos en revistas fuera de Brasil

\begin{tabular}{l|l|l}
\hline Países / Títulos de las revistas & Totalidad & Artículos \\
\hline $\begin{array}{l}\text { ARGENTINA - E+E Estudios de Extensión en Humanidades }(n=2) \text { Estudios } \\
\text { Rurales; Miríada: Investigaciones en Ciencias Sociales; Praxis Educativa; Red } \\
\text { Sociales; REMIE - Revista Multidisciplinar de Investigación Educativa; Revista de la } \\
\text { Escuela de Ciencias de la Educación; Revista del IICE }\end{array}$ & 8 & 9 \\
\hline $\begin{array}{l}\text { ESPAÑA - Enseñanza de las Ciencias }(n=2) ; \text { Educar-Barcelona; Historia y } \\
\text { Memoria de la Educación; Revista Interuniversitaria de Formación del }\end{array}$ & 5 & 6 \\
$\begin{array}{l}\text { Profesorado; Spanish Journal of Psychology } \\
\text { COLOMBIA - Avances en Psicología Latinoamericana; Cuadernos de Desarrollo }\end{array}$ & 4 & 4 \\
\hline $\begin{array}{l}\text { Rural; Revista Latinoamericana de Etnomatemática; Revista NuestrAmérica } \\
\text { de Investigación Educativa; Revista del Centro de Investigación; Revista Mexicana } \\
\text { de Investigación Educativa }\end{array}$ & 4 & 5 \\
\hline $\begin{array}{l}\text { FRANCIA - Revue Française de Pédagogie }(n=2) ; \text { Questions Vives: Recherches en } \\
\text { Education; Recherches et Éducations }\end{array}$ & 3 & 4
\end{tabular}




\begin{tabular}{l|l|l}
$\begin{array}{l}\text { PORTUGAL - Revista Portuguesa de Educação; Revista UIIPS_Instituto Politérico } \\
\text { de Santarém; Sísifo: Revista de Ciências da Educação }\end{array}$ & 3 & 3 \\
\hline $\begin{array}{l}\text { CANADÁ - Éducation et Francophonie; Revue Internationale de Pédagogie de } \\
\text { I'Enseignement Supérieur }\end{array}$ & 2 & 2 \\
\hline $\begin{array}{l}\text { CHILE - Cisma: Revista del Centro Telúrico de Investigaciones Teóricas; REXE - } \\
\text { Revista de Estudios y Experiencias en Educación }\end{array}$ & 2 & 2 \\
\hline PERÚ - Educación; Revista de Investigación Apuntes Psicológicos & 2 & 2 \\
\hline CHINA - International Journal of Current Science and Technology & 1 & 1 \\
\hline ITALIA - Studi sulla Formazione & 1 & 1 \\
\hline VENEZUELA - Espacios & 1 & 2 \\
\hline REINO UNIDO - Gender and Education & 1 & 1 \\
\hline Totalidad & $\mathbf{3 7}$ & $\mathbf{4 2}$ \\
\hline
\end{tabular}

También se analizó la clasificación de revistas en la lista preliminar Qualis/Capes. Este listado, elaborado por la Coordinación para el Perfeccionamiento del Personal de Educación Superior (CAPES, 2019), retrata el producto intelectual de los programas de posgrado brasileños y es utilizado por este organismo para clasificar la calidad de la producción científica en todas las áreas del conocimiento. Según esta metodología, las revistas reciben una puntuación que resulta en una clasificación de nueve estratos ( $A 1, A 2, A 3, A 4, B 1, B 2, B 3, B 4$ y C) y aquellos que no fueron puntuados (NP).

La Figura 4 muestra la clasificación de revistas $(n=157)$ según la lista preliminar Qualis/Capes. Se observa que el 57\% $(n=143)$ de los artículos fue publicado en el $49 \%(n=77)$ de las revistas que obtuvieron clasificación en los estratos más altos de la lista Qualis/Capes, es decir, entre A1 y A4, demostrando que la producción de investigación científica sobre Pedagogía de la Alternancia ha tenido éxito en cuanto a la aceptación para su publicación en estas revistas.

\section{Figura 4}

Distribución de los periódicos de acuerdo con la clasificación Qualis/Capes

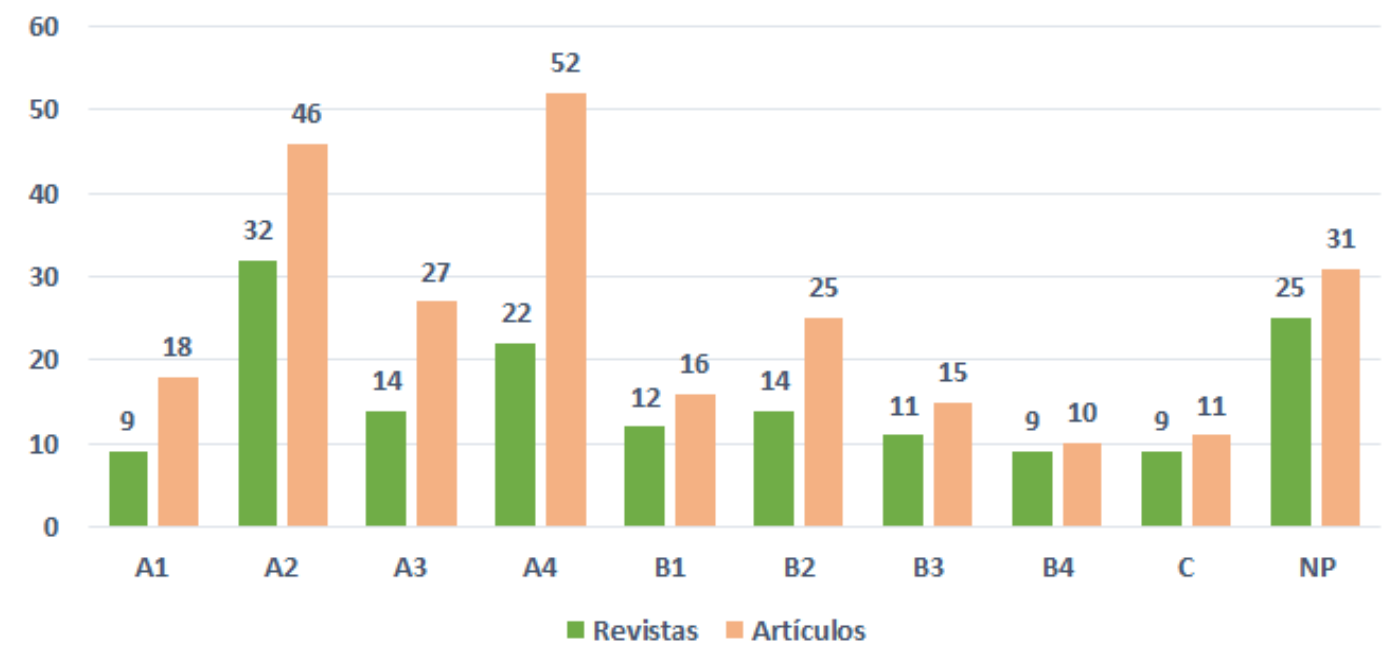


Además, la Figura 4 muestra un expresivo contingente, representado por el 29,3\% $(n=46)$ de los títulos de revistas clasificadas en los estratos entre B1 y B4, que divulgaron el $26,3 \%$ ( $n=66$ ) de los artículos. Por último, el 21,7\% (n = 34) de las revistas clasificadas en los estratos más bajos, es decir, como C o NP (no puntuado), publicaron un porcentaje del 16,7\% ( $n=42$ ) de artículos.

La lectura integral de los artículos $(n=251)$ permitió identificar los tipos de estudios realizados, de acuerdo con las siguientes categorizaciones:

estudio de caso: se caracteriza por ser una investigación empírica que busca en profundidad un fenómeno contemporáneo en un contexto del mundo real. Se pueden clasificar como estudios de caso único o múltiples, como explica Yin (2015).

Ensayo teórico, también denominado ensayo académico o artículo teórico. Para Leal (2011), el propósito del ensayo radica en proponer preguntas y examinar aporías, sin una organización preestablecida y, muchas veces, con carácter subjetivo. A menudo, el ensayo es una reflexión sobre el presente, aunque su autor/a busca apoyo en el pasado para comprender mejor los problemas contemporáneos. Según Michel (2015), el ensayo académico defiende una idea o visión original y puede presentar un nuevo enfoque o problema del objeto de interés. En el Manual de publicación de la American Psychological Association (APA, 2012), el artículo teórico es un tipo de texto en el que los autores trazan el desarrollo de la teoría para expandir y refinar los constructos, presentar una nueva teoría o analizar una teoría existente, señalando fallas o demostrando la ventaja de una teoría sobre la otra.

Metanálisis: según el Manual de publicaciones de la APA (2012), las revisiones de la literatura, incluidas las síntesis de investigación y los metanálisis, son evaluaciones críticas de material que ya se ha publicado. En los metanálisis, los/as autores/as utilizan procedimientos cuantitativos para combinar estadísticamente los resultados de los estudios. Para Hayashi y Gonçalves (2016), los metanálisis suelen utilizarse como sinónimo de "estado del arte", sin embargo, su significado más preciso y original es describir y analizar cuantitativa y cualitativamente las síntesis de investigación consolidadas en los artículos que componen un corpus de análisis dado.

A partir de esta categorización de los artículos analizados $(n=251)$, los resultados revelaron la preponderancia de estudios de caso $(n=183)$, seguidos de ensayos teóricos $(n=60)$ y metanálisis $(n=8)$. La Figura 5 presenta estos resultados considerando el período entre 1981 y 2019, cuando se publicaron los artículos. 


\section{Figura 5}

Distribución temporal de los tipos de estudios de los artículos

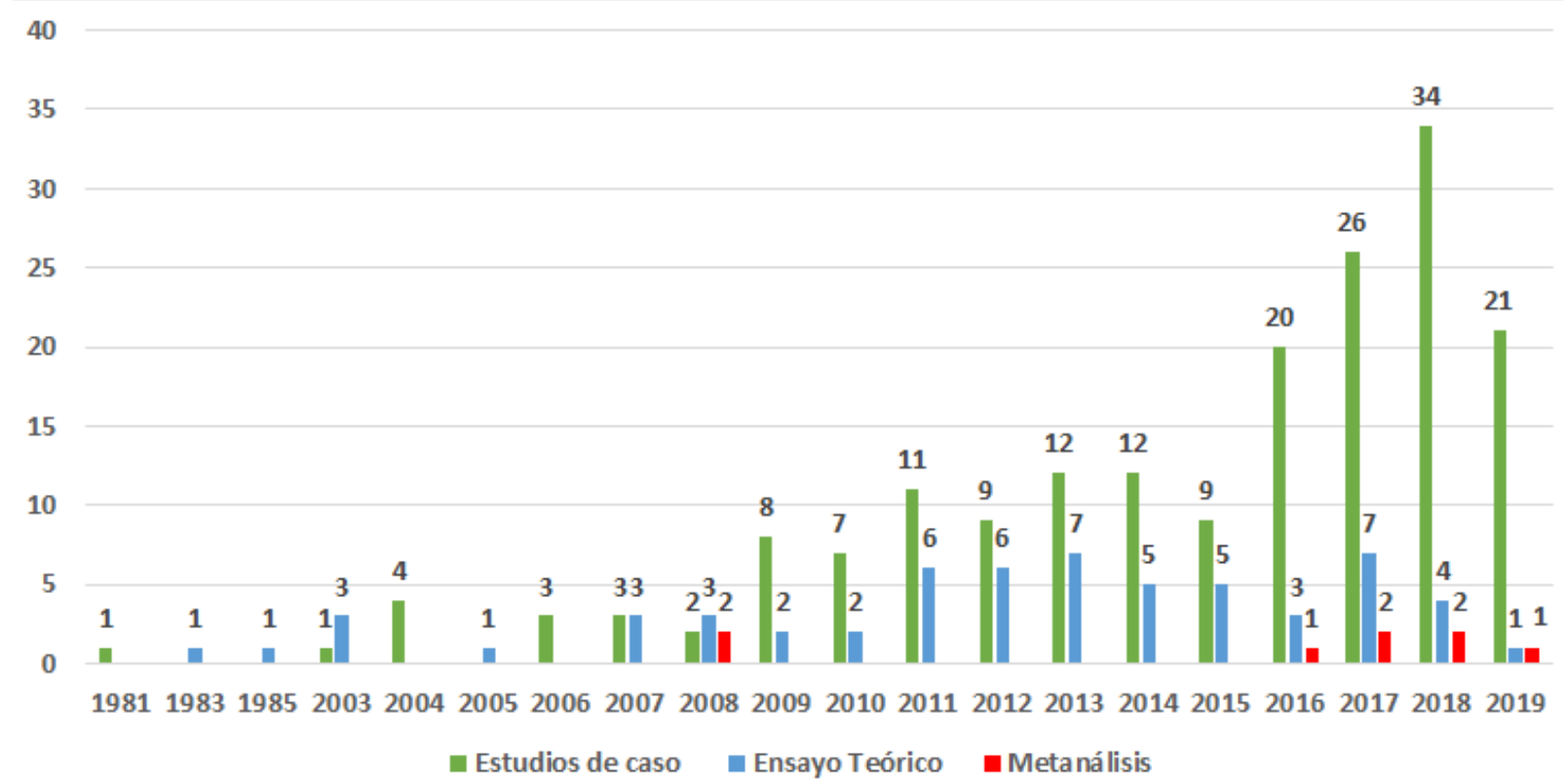

Se puede observar, en la Figura 5, que, entre 1981 y 2008, los números de artículos "estudio de caso" ( $n=14)$ y "ensayo teórico" $(n=12)$ se mantienen estables con pocas variaciones. De 2009 a 2015, hay un aumento de ambos tipos, con un promedio anual del 7,5 y un incremento en las puntuaciones de los "estudios de caso" ( $n=68)$ y los "ensayos teóricos" $(n=33)$. El crecimiento más expresivo en los "estudio de caso" ( $n=101)$ se da entre 2016 y 2019, sin embargo, en este período, hay una disminución importante en los "ensayos teóricos" ( $n=15)$, lo que puede sugerir alguna saturación de este tipo de estudio y denota la ausencia de nuevos enfoques y constructos teóricos sobre la "Pedagogía de la Alternancia".

En relación con los artículos ( $n=183$ ) del tipo "estudios de caso", se investigaron los loci donde se llevaron a cabo las investigaciones. La Figura 6 muestra los resultados obtenidos según las regiones y municipios brasileños y del exterior cuando se trata de investigaciones realizadas fuera de Brasil. 


\section{Figura 6}

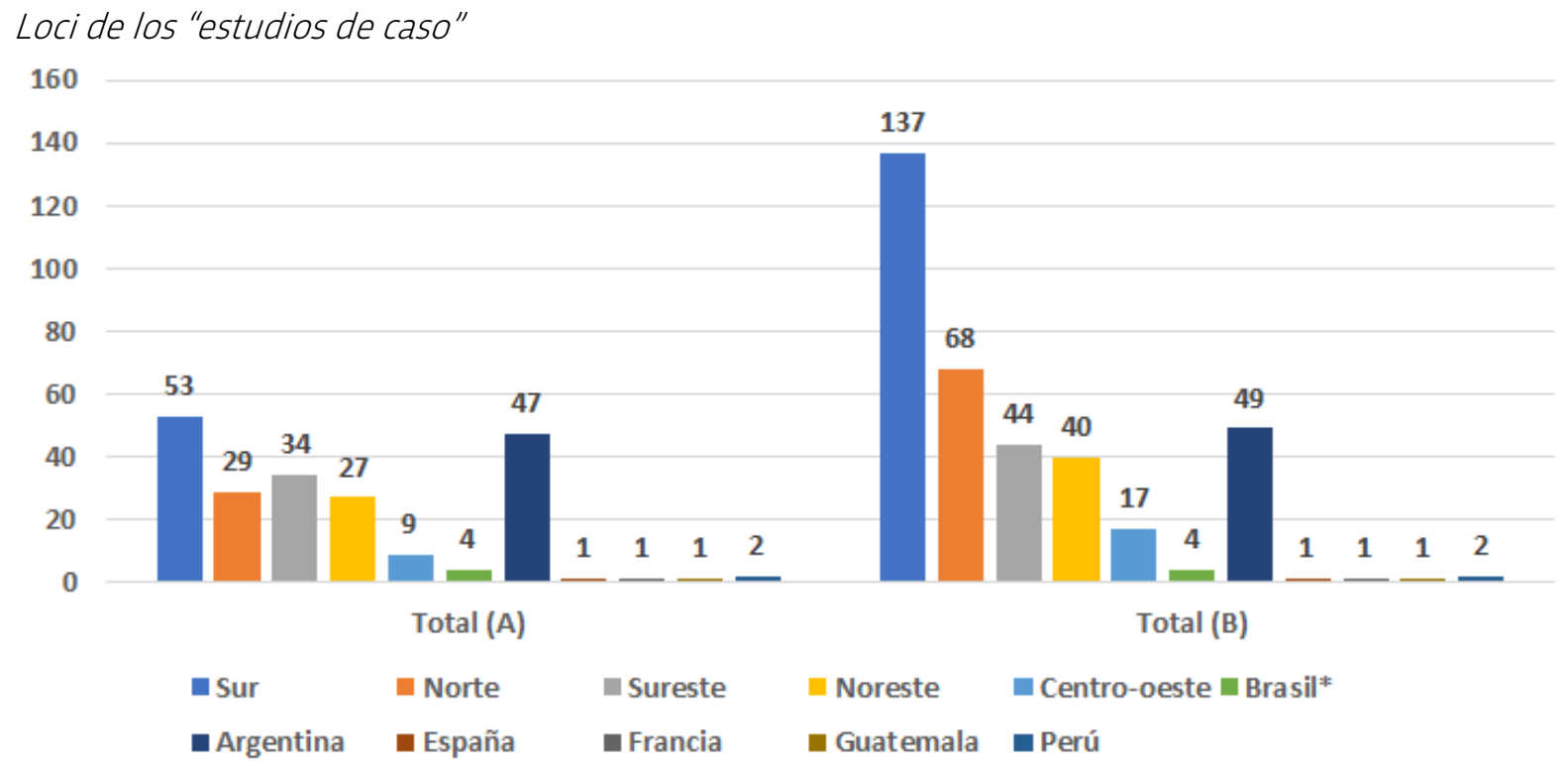

*Brasil: estudios que no identificaron el municipio.

En la Figura 6, se puede observar que el Total $(A)(n=364)$ de los municipios y países enfocados en los "estudios de caso" $(n=183)$ es superior al Total $(B)(n=208)$. Esto se debe a que el mismo municipio puede haber sido objeto de diferentes "estudios de caso" y a que existen estudios de este tipo que cubren más de un municipio, como ocurre con los "estudios de caso múltiples".

Así, considerando el Total (A), Brasil lidera con el 75\% ( $n=156)$ de los municipios, que se distribuyen en las cinco regiones del país. Luego, aparecen municipios de Argentina con 22,6\% ( $n$ $=47)$ y Perú, con $1 \%(n=2)$. Los municipios de España $(n=1)$, Francia $(n=1)$ y Guatemala $(n=1)$ juntos representaron el $1,4 \%(n=3)$ de los objetos en los "estudios de caso". A continuación, la Tabla 3 muestra la distribución de estas ubicaciones en Brasil y en el exterior.

\section{Tabla 3}

Municipios investigados en los estudios de caso

\begin{tabular}{l|l|l}
\hline Regiones & Estado/Países & Municipios \\
\hline \multirow{3}{*}{$\begin{array}{l}\text { SUR } \\
(n=53)\end{array}$} & $\begin{array}{l}\text { Paraná } \\
(n=28)\end{array}$ & $\begin{array}{l}\text { Machado; Dois Vizinhos; Enéas Marques; Francisco Beltrão; Irati; Lapa; } \\
\text { Manfrinópolis; Marmeleiro; Matinhos, Nova Prata do Iguaçu; Paraná*; Pato } \\
\text { Branco; Pérola do Oeste; Pinhão; Porto Barreiro; Realeza; Reserva; Santa } \\
\text { Izabel d'Oeste; Santa Maria d'Oeste; Santo Antônio do Sudoeste; São Jorge } \\
\text { d'Oeste; Sapopema; Sulina. }\end{array}$ \\
\cline { 2 - 3 } & $\begin{array}{l}\text { Santa Catarina } \\
(n=9)\end{array}$ & $\begin{array}{l}\text { Campos Novos; Chapecó; Dionísio Cerqueira; Florianópolis; Imbituba; } \\
\text { Quilombo; Riqueza; São Francisco do Sul; São José do Cedro. }\end{array}$ \\
\hline
\end{tabular}


Estudio bibliométrico sobre la Pedagogía de la Alternancia en artículos científicos (1981-2019). I María Cristina Piumbato Innocentini Hayashi; Alexandre Masson Maroldi; Taísa Grasiela Gomes Liduenha Gonçalves; Carlos Roberto Massao Hayashi.

\begin{tabular}{|c|c|c|}
\hline & $\begin{array}{l}\text { Rio Grande do } \\
\text { Sul } \\
(n=16)\end{array}$ & $\begin{array}{l}\text { Canoinhas; Catuípe, Cidreira; Dilermando de Aguiar; Dom Pedrito, Erechim; } \\
\text { Frederico Westphalen; Jaguari; Mata; Nova Esperança do Sul; Porto Alegre; } \\
\text { Rio Grande; Santa Cruz do Sul; Sertão; Tramandaí; Veranópolis }\end{array}$ \\
\hline \multirow{5}{*}{$\begin{array}{l}\text { NORTE } \\
(n=29)\end{array}$} & Acre $(n=1)$ & Acrelândia \\
\hline & Amapá $(n=6)$ & Amapá*; Macapá; Mazagão, Pacui; Pedra Branca do Amaparí; Tartarugalzinho \\
\hline & Pará $(n=8)$ & Altamira; Belém; Belterra; Cametá; Marabá; Portel; Santarém; Uruará \\
\hline & $\begin{array}{l}\text { Rondônia ( } n= \\
10)\end{array}$ & $\begin{array}{l}\text { Buritis; Cacoal; Candeias do Jamaeri; Colorado d'Oeste; Costa Marques; } \\
\text { Guajará Mirim; Jaru; Ji-Paraná; Novo Horizonte d'Oeste; Vale do Paraíso }\end{array}$ \\
\hline & $\begin{array}{l}\text { Tocantins }(n= \\
\text { 4) }\end{array}$ & Colinas do Tocantins; Esperantina; Porto Nacional; Tocantinópolis \\
\hline \multirow{4}{*}{$\begin{array}{l}\text { SURESTE } \\
(n=34)\end{array}$} & $\begin{array}{l}\text { Espírito Santo }(\mathrm{n} \\
=11)\end{array}$ & $\begin{array}{l}\text { Águia Branca; Barra de São Francisco; Castelo; Colatina; Goiabeiras; Jaguaré; } \\
\text { Rio Novo do Sul;Santa Maria de Jetibá; São Mateus; São Gabriel de Palha; } \\
\text { Vitória }\end{array}$ \\
\hline & $\begin{array}{l}\text { Minas Gerais ( } \mathrm{n} \\
=12)\end{array}$ & $\begin{array}{l}\text { Acaiaca, Almenara; Araponga; Belo Horizonte; Ervália; Inconfidentes; Itaobim; } \\
\text { Januária; Natalândia; Veredinha; Viçosa; Virgem da Lapa; }\end{array}$ \\
\hline & $\begin{array}{l}\text { Rio de Janeiro }(\mathrm{n} \\
=1)\end{array}$ & Seropédica \\
\hline & $\begin{array}{l}\text { São Paulo }(n= \\
\text { 10) }\end{array}$ & $\begin{array}{l}\text { Andradina; Bananal; Maracaí; Mirante do Paranapanema; Peruíbe; } \\
\text { Piratininga; Presidente Venceslau; Promissão; Rancharia; São Lourenço do } \\
\text { Sul }\end{array}$ \\
\hline \multirow{5}{*}{$\begin{array}{l}\text { NORESTE } \\
(n=27)\end{array}$} & Bahia $(n=16)$ & $\begin{array}{l}\text { Angical; Amargosa, Bahia*; Brotas de Macaúbas; Correntina; Cruz das Almas; } \\
\text { Irará; Jaguaquara; Monte Santo; Quixabeira, Rio Real; Salvador, Santa Inês; } \\
\text { Semiárido da Bahia*; Sobradinho; Valente }\end{array}$ \\
\hline & Ceará $(n=2)$ & Crato; Independência \\
\hline & $\begin{array}{l}\text { Maranhão }(n= \\
\text { 4) }\end{array}$ & Babacal; Balsas; São Luís; Timon \\
\hline & Piauí $(n=2)$ & Floriano; Teresina \\
\hline & Sergipe $(n=3)$ & Japoatã; Ladeirinhas; Sergipe* \\
\hline \multirow{4}{*}{$\begin{array}{l}\text { CENTRO- } \\
\text { OESTE } \\
(n=9)\end{array}$} & $\begin{array}{l}\text { Distrito Federal } \\
(\mathrm{n}=2)\end{array}$ & Brasília; Planaltina \\
\hline & Goiás $(n=3)$ & Goiás; Orizona; Uirapuru \\
\hline & $\begin{array}{l}\text { Mato Grosso do } \\
\text { Sul }(n=3)\end{array}$ & Aquidauana; Campo Grande; Sidrolândia \\
\hline & $\begin{array}{l}\text { Mato Grosso ( } \mathrm{n} \\
=1)\end{array}$ & Terra Nova do Norte \\
\hline Brasil & $(n=4)$ & Sin identificación del municipio \\
\hline $\begin{array}{l}\text { PAÍSES } \\
(n=52)\end{array}$ & $\begin{array}{l}\text { Argentina } \\
(n=47)\end{array}$ & $\begin{array}{l}9 \text { de Julio; Azul; Balcarce; Bragado; Buenos Aires; Cañeulas; Carlos Tejedor; } \\
\text { Carmen de Patagones; Chaco; Córdoba, Coronel Brandsen; Coronel Pringles; } \\
\text { Corrientes; Daireaux; Dorrego; El Billar; Exaltación de la Cruz; Ceneral } \\
\text { Alvarado; General Belgrano; General de Lamadrid; General Guido; General } \\
\text { Viamonte; González Chaves; Guaminí; Las Flores; Lavalle; Lobos; Magdala; } \\
\text { Magdalena; Mercedes; Misiones; Olavarría; Puán; Rauch; Reconquista; Rojas; }\end{array}$ \\
\hline
\end{tabular}




\begin{tabular}{l|l|l}
\hline & $\begin{array}{l}\text { Saladillo; San Andrés de Giles; San Cayetano; San Pedro; Santa Fé; San } \\
\text { Ignacio; Santiago del Estero; Salta; Tornquist; Trenque Lauquen; Tres Lomas }\end{array}$ \\
\cline { 2 - 3 } & España $(\mathrm{n}=1)$ & Valencia \\
\cline { 2 - 3 } & Francia $(\mathrm{n}=1)$ & Angers \\
\cline { 2 - 3 } $\begin{array}{l}\text { Guatemala }(\mathrm{n}= \\
1)\end{array}$ & Quetzaltenango \\
\cline { 2 - 3 } & Perú $(\mathrm{n}=2)$ & Cuzco; Piura \\
\hline
\end{tabular}

*Solo el Estado o la región fueron mencionados en el estudio.

El análisis de los artículos del tipo "estudios de caso" publicados en Brasil y en el exterior permitió categorizar los objetos de estudio de esas investigaciones de acuerdo con la siguiente clasificación: Escuelas Familiares Agrícolas (EFAs), Maison Familiale Rurale (MFR), Centros Familiares de Formación en Alternancia (CEFFAs), Centros Educativos para la Producción Total (CEPT), Centro Rural de Formación en Alternancia (CRFA) y Casas Familiares Rurales (CFRs), así como cursos superiores de licenciatura en Educación del Campo, cursos técnicos y de especialización, instituciones de enseñanza, comunidades y programas gubernamentales que adoptan la Pedagogía de la Alternancia como metodología (Figura 7). Cabe señalar que el total de objetos de estudio $(n=240)$ es mayor que el total de estudios de caso $(n=183)$, ya que algunos de estos pueden haber abordado varios objetos de estudio.

Los resultados también señalaron que la mayoría $(n=221)$ de los objetos de estudio se refieren a estudios de caso realizados en Brasil. Por otro lado, los estudios de caso realizados en el extranjero reunieron el $7,91 \%(n=19)$ de la totalidad de los objetos de estudio.

\section{Figura 7}

Objetos de estudio de los "estudios de caso" realizados en Brasil y exterior

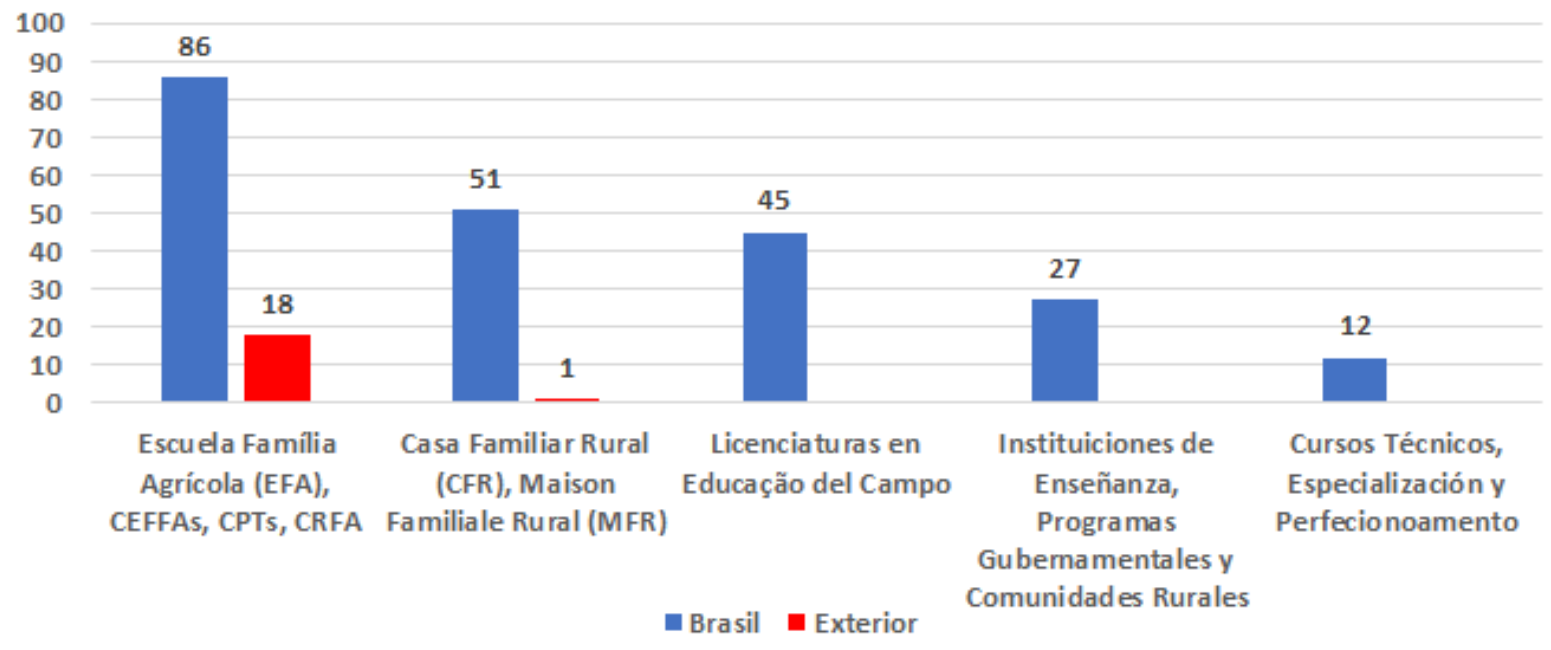

Las EFAs fueron los objetos de estudio $(n=104)$ que predominaron en los estudios de caso, con la mayoría $(n=86)$ ubicados en Brasil y, los demás, en Argentina $(n=11)$ y España $(n=1)$. 
Entre las EFAs de Brasil, predominan las del norte $(n=37)$, seguidas de las del noreste $(n=21)$ y sureste $(n=15)$.

Con menor incidencia, surgen las EFAs de las regiones sur y centro-oeste, con el mismo número cada una $(n=6)$. A su vez, la puntuación $(n=19)$ de las EFAs, CFRs, CEPTs y CRFAs del exterior fueron objetos de estudio minoritarios. La Tabla 4 presenta estos datos distribuidos por estados y regiones de Brasil y países del exterior.

\section{Tabla 4}

Distribución de los objetos de estudio EFAs, CEFFA, CEPTS y CRFAs

\begin{tabular}{|c|c|c|c|}
\hline Escuelas Familias Agrícolas (EFA) & Estado/País & Región & Estudios \\
\hline EFA Jean Pierre Mingan & Acre & \multirow{4}{*}{$\begin{array}{l}\text { Norte } \\
(n=37)\end{array}$} & 2 \\
\hline $\begin{array}{l}\text { EFA do Cedro; EFA do Carvão; EFA do Maracá; EFA do Pacuí }(n=2) ; \\
\text { EFA Perimetral Norte }\end{array}$ & Amapá & & 6 \\
\hline $\begin{array}{l}\text { EFA Antonio Possomai }(n=2) \text {; EFA Chico Mendes }(n=2) \text {; EFA Dom } \\
\text { Orione }(n=2) \text {; EFA Flor do Amazonas }(n=2) \text {; EFA Itapirema }(n=2) \text {; } \\
\text { EFA Manoel Ribeiro }(n=2) \text {; EFA Pe. Ezequiel Ramin }(n=2) \text {; EFA Vale } \\
\text { do Guaporé }(n=2) \text {; EFA Vale do Paraíso }(n=2) \text {; CEFFA de Ji-Paraná } \\
(n=3)\end{array}$ & Rondônia & & 21 \\
\hline $\begin{array}{l}\text { EFA de Porto Nacional }(n=4) ; \text { EFA de Tocantins }(n=1) ; \text { EFA "Zé de } \\
\text { Deus" }(n=3)\end{array}$ & Tocantins & & 8 \\
\hline $\begin{array}{l}\text { EFA Avani de Lima Cunha }(n=2) \text {; EFA do Sertão }(n=5) \text {; EFA de } \\
\text { Quixabeira }(n=1) \text {; EFA José Nunes da Matta }(n=2) \text {; EFAs da Bahia* } \\
(n=1) \text {; EFAs do Semiárido Baiano* }(n=1)\end{array}$ & Bahia & \multirow{5}{*}{$\begin{array}{l}\text { Noreste } \\
(n=21)\end{array}$} & 12 \\
\hline EFA Dom Fragoso $(n=5)$ & Ceará & & 5 \\
\hline EFA Rio Peixe $(n=1)$ & Maranhão & & 1 \\
\hline EFA Baixão do Carlos $(n=1)$ & Piauí & & 1 \\
\hline EFA Ladeirinhas $(n=2)$ & Sergipe & & 2 \\
\hline $\begin{array}{l}\text { EFA de Goiás }(n=2) \text {; EFA de Orizona }(n=1) \text {; EFA de Uirapuru }(n=1) \text {; } \\
\text { EFA do Bico do Papagaio Padre Josimo }(n=1)\end{array}$ & Goiás & \multirow{2}{*}{$\begin{array}{l}\text { Centro-Oeste } \\
(n=6)\end{array}$} & 5 \\
\hline EFA de Sidrolândia & $\begin{array}{l}\text { Mato Grosso } \\
\text { do Sul }\end{array}$ & & 1 \\
\hline $\begin{array}{l}\text { EFA de Castelo; EFA de Nestor Comes; EFA de São João do Garrafão; } \\
\text { EFA de Rio Novo do Sul; EFA do Bley }\end{array}$ & $\begin{array}{l}\text { Espirito } \\
\text { Santo }\end{array}$ & \multirow{2}{*}{$\begin{array}{l}\text { Sureste } \\
(n=15)\end{array}$} & 5 \\
\hline $\begin{array}{l}\text { EFA de Natalândia }(n=1) \text {; EFA Paulo Freire de Acaiaca }(n=2) \text {; EFA } \\
\text { Puris de Araponga }(n=3) \text {; EFA Serra do Brigadeiro }(n=1) ; \text { EFA de } \\
\text { Veredinha }(n=1) ; \text { EFA Virgem da Lapa }(n=1) ; \text { EFA Bontempo }(n=1) ;\end{array}$ & Minas Gerais & & 10 \\
\hline EFA de Santa Cruz do Sul & $\begin{array}{l}\text { Rio Grande } \\
\text { do Sul }\end{array}$ & $\operatorname{Sur}(n=6)$ & 6 \\
\hline $\begin{array}{l}\text { EFA de Buenos Aires }(n=1) \text {; EFA de Córdoba }(n=1) \text {; EFAde Corrientes } \\
(n=1) ; \text { EFA de Santiago del Estero }(n=1) ; \text { EFA de Salta }(n=1) \text {; EFAs de } \\
\text { Santa Fé }(n=1) \text {; EFAs de San Pedro e Misiones }(n=2) \text {; EFA San }\end{array}$ & \multicolumn{2}{|l|}{ Argentina } & 11 \\
\hline
\end{tabular}




\begin{tabular}{l|l|l}
\hline $\begin{array}{l}\text { Ignacio }(n=1) ; \text { Escuela Campesina de Agroecologia No } 502(n=1) ; \\
\text { EFAN8202 de Moussy }(n=1)\end{array}$ & & \\
\hline $\begin{array}{l}\text { Escuela de Formació Agrícola Comarcal La Serranía (Chulilla) en } \\
\text { Valencia }(n=1)\end{array}$ & España & 1 \\
\hline CEFFA, CPTs. CRFs & & \\
\hline Centros Familiares de Formación en Alternancia-CEFFAs & Brasil & 1 \\
\hline $\begin{array}{l}\text { Centros Educativos para la Producción Total (CEPT) }(n=1) ; \text { Centro } \\
\text { Educativo Isauro Arancibia }(n=1) ; \text { Centros Educativos para la } \\
\text { Producción Total - CEPT No 5 en Miranda }(n=1)\end{array}$ & Argentina & 3 \\
\hline $\begin{array}{l}\text { Centro Rural de Formación en Alternancia de Occopata - Cuzco }(n= \\
\text { 1); Centros Rurales de Formación por Alternancia }(n=1)\end{array}$ & Perú & \\
\hline Centros Rurales de Formación por Alternancia $(n=1)$ & Guatemala & 2 \\
\hline Total & & 1 \\
\hline
\end{tabular}

La Tabla 5, a continuación, presenta los resultados de los estudios de caso que eligieron a las Casas Familiares Rurales (CFR) de Brasil y del exterior como objeto de estudio. Estos resultados indican que, con el 90,2\% ( $n=46)$, prevalecieron los CFRs ubicados en el sur $(n=46)$ del país, con mayor incidencia para los de Paraná $(\mathrm{n}=36)$. En el exterior, solo la Maison Familiale Rurale (MFR) ubicada en Angers (Francia) fue objeto de un estudio de caso.

\section{Tabla 5}

Distribución de los objetos de estudio CFR y MFR en Brasil y Francia

\begin{tabular}{|c|c|c|c|}
\hline Casas Familiares Rurales (CFR) y Maison Familiale Rurale (MFR) & Estado/País & Región & Estudios \\
\hline $\begin{array}{l}\text { CFR de Altamira; CFR de Belterra. CFR de Cametá; CFR de Santarém; CFR de } \\
\text { Uruará }\end{array}$ & Pará & Norte & 5 \\
\hline $\begin{array}{l}\text { CFR da Lapa; CRF de Bituruna; CRF de Chopinzinho; CRF de Coronel Vivida ( } n= \\
\text { 4); CRF de Cruz Machado; CRF de Enéas Marques; CRF de Francisco Beltrão; } \\
\text { CRF de Manfrinópolis }(n=3) ; C R F \text { de Marmeleiro }(n=2) ; C R F \text { de Nova Prata } \\
\text { do Iguaçu; CRF de Pato Branco }(n=5) \text {; CRF de Pinhão; CRF de Porto Barreiro; } \\
\text { CRF de Realeza }(n=2) ; C R F \text { de Reserva; CRF de Santa Izabel do Oeste; CRF de } \\
\text { Santa Maria do Oeste; CRF de Santo Antônio do Sudoeste; CRF Padre Sasaki; } \\
\text { CRF de Sulina; CFRs do Sudoeste do Paraná* }(n=4) ; C F R s \text { de Paraná* }\end{array}$ & Paraná & Sur & 36 \\
\hline $\begin{array}{l}\text { CFR de Quilombo; CFR de Riqueza; CFR de Santo Agostinho }(n=2) \text {; CFR de São } \\
\text { José do Cedro; CFR do Mar de São Francisco do Sul }\end{array}$ & $\begin{array}{l}\text { Santa } \\
\text { Catarina } \\
\end{array}$ & & 6 \\
\hline $\begin{array}{l}\text { CFR de Santo Isidoro; CFR Francisco Westphalen; CFR de Três Vendas; CFR do } \\
\text { Vale do Jaguari }\end{array}$ & $\begin{array}{l}\text { Rio Grande } \\
\text { do Sul }\end{array}$ & & 4 \\
\hline Maison Familiale Rurale (MFR) - Angers & Francia & & 1 \\
\hline Total & & & 52 \\
\hline
\end{tabular}

*CFR sin identificación individual. 
Los cursos de educación superior en Educación del Campo $(n=43)$ y Etnometodología ( $n$ = 1) que adoptaron la perspectiva de la Pedagogía de la Alternancia se distribuyen entre las universidades $(n=44)$ y el Instituto Federal de Educación $(n=1)$ ubicados en las regiones norte ( $n$ $=14)$, sureste $(n=10)$, sur $(n=10)$, noreste $(n=6)$ y centro-oeste $(n=4)$ de Brasil, como se muestra en los datos de la Tabla 6.

\section{Tabla 6}

Instituciones que ofrecen licenciaturas con enfoque en Pedagogía de la Alternancia

\begin{tabular}{|c|c|c|c|}
\hline Instituciones & Estado & Región & Estudios \\
\hline Universidade Federal do Espírito Santo & Espírito Santo & \multirow{5}{*}{$\begin{array}{l}\text { Sureste } \\
(n=10)\end{array}$} & 2 \\
\hline $\begin{array}{l}\text { Instituto Federal de Educação, Ciência e Tecnologia do Sul de } \\
\text { Minas Gerais }\end{array}$ & \multirow{3}{*}{ Minas Gerais } & & 1 \\
\hline Universidade Federal de Viçosa & & & 4 \\
\hline Universidade Federal de Minas Gerais & & & 1 \\
\hline Universidade Federal Rural do Rio de Janeiro & Rio de Janeiro & & 2 \\
\hline Universidade Federal do Amapá & Amapá & \multirow{5}{*}{$\begin{array}{l}\text { Norte } \\
(n=14)\end{array}$} & 1 \\
\hline Universidade Federal do Sul e Sudeste do Pará & \multirow{3}{*}{ Pará } & & 4 \\
\hline Instituto Federal de Educação e Tecnologia do Pará & & & 4 \\
\hline Universidade Federal do Pará (Etnomedología) & & & 2 \\
\hline Universidade Federal de Tocantins & Tocantins & & 3 \\
\hline Universidade Federal de Santa Catarina & Santa Catarina & \multirow{5}{*}{$\begin{array}{l}\text { Sur } \\
(n=10)\end{array}$} & 3 \\
\hline Universidade Federal da Fronteira do Sul & \multirow{4}{*}{ Rio Grande do Sul } & & 3 \\
\hline Universidade Federal do Rio Grande & & & 1 \\
\hline Universidade Federal do Rio Grande do Sul & & & 2 \\
\hline Universidade Federal do Pampa & & & 2 \\
\hline Universidade Federal do Recôncavo da Bahia & Bahia & \multirow{4}{*}{$\begin{array}{l}\text { Noreste } \\
(n=6)\end{array}$} & 2 \\
\hline Universidade Regional do Cariri & Ceará & & 1 \\
\hline Universidade Federal do Maranhão & Maranhão & & 2 \\
\hline Universidade Federal do Piauí & Piauí & & 1 \\
\hline Universidade Federal de Mato Grosso do Sul & $\begin{array}{l}\text { Mato Grosso do } \\
\text { Sul }\end{array}$ & \multirow{3}{*}{$\begin{array}{l}\text { Centro- } \\
\text { Oeste } \\
(n=4)\end{array}$} & 1 \\
\hline Universidade Federal de Goiás & \multirow{2}{*}{ Goiás } & & 1 \\
\hline Universidade de Brasília & & & 2 \\
\hline Total & & & 45 \\
\hline
\end{tabular}

Los estudios de caso también eligieron cursos técnicos y de perfeccionamiento como objetos de estudio que adoptan la perspectiva de la PA, como se muestra en los datos de la Tabla 7.

La mayoría de estos son cursos técnicos en Agropecuaria y Agroecología $(n=6)$, además de cursos de perfeccionamiento $(n=2)$ y especialización $(n=1)$, que se ofrecen a profesionales y 
docentes de la educación, así como en la modalidad Educación de Jóvenes y Adultos (EJA).

También se observa que solo en el Norte del país no se presentaron estudios de caso con estos objetos de estudio.

\section{Tabla 7}

Cursos técnicos, de perfeccionamiento y especialización con perspectiva PA

\begin{tabular}{|c|c|c|c|}
\hline Cursos & Estado & Región & Estudios \\
\hline $\begin{array}{l}\text { Curso Técnico en Agropecuaria con Énfasis en Agroecología Integrado a } \\
\text { la Enseñanza Secundaria del Instituto Federal de Educación, Ciencia y } \\
\text { Tecnología del Rio Grande do Sul e Instituto Educar }\end{array}$ & \multirow{2}{*}{$\begin{array}{l}\text { Rio } \\
\text { Grande } \\
\text { do Sul }\end{array}$} & \multirow{2}{*}{ Sur } & 2 \\
\hline $\begin{array}{l}\text { Curso de Especialización en Educación Profesional Integrada a la } \\
\text { Educación Básica en la Modalidad Educación de Jóvenes y Adultos - } \\
\text { Propuesta diferenciada para Indígenas }\end{array}$ & & & 1 \\
\hline $\begin{array}{l}\text { Curso Técnico en Agropecuaria en el Instituto Federal de Educación, } \\
\text { Ciencia y Tecnología de Brasília - Campus de Planaltina }\end{array}$ & \multirow[b]{2}{*}{$\begin{array}{l}\text { Distrito } \\
\text { Federal }\end{array}$} & \multirow[b]{2}{*}{$\begin{array}{l}\text { Centro- } \\
\text { Oeste }\end{array}$} & 2 \\
\hline $\begin{array}{l}\text { Cursos de Educación del Campo de la Escuela de Perfeccionamiento de } \\
\text { los Profesionales de la Educación (EAPE) de la Secretaría de Estado de } \\
\text { Educación del Distrito Federal }\end{array}$ & & & 2 \\
\hline $\begin{array}{l}\text { Curso Técnico de Agropecuaria, del Instituto Federal del Espírito Santo } \\
\text { - Campus Barra de São Francisco }\end{array}$ & $\begin{array}{l}\text { Espírito } \\
\text { Santo }\end{array}$ & \multirow{2}{*}{ Sureste } & 1 \\
\hline $\begin{array}{l}\text { Curso Técnico en Agropecuaria - Modalidad Alternancia, de la Escuela } \\
\text { Técnica Estadual (ETEC) "Sebastiana Augusta de Moraes" en Andradina }\end{array}$ & $\begin{array}{l}\text { São } \\
\text { Paulo }\end{array}$ & & 1 \\
\hline $\begin{array}{l}\text { Curso Técnico en Agropecuaria Integrado a la Enseñanza Secundaria en } \\
\text { la Modalidad de Educación de Jóvenes y Adultos/Proeja en la } \\
\text { Metodología de la Alternancia en el campus Santa Inês en el Instituto } \\
\text { Federal Baiano - Bahia }\end{array}$ & Bahia & \multirow{3}{*}{ Noreste } & 1 \\
\hline $\begin{array}{l}\text { Curso de Perfeccionamiento de Profesores del PRONACAMPO - Escuela } \\
\text { de la Tierra de Sergipe }\end{array}$ & Sergipe & & 1 \\
\hline $\begin{array}{l}\text { Curso Técnico en Agroecología del Campus Rural de Marabá, del } \\
\text { Instituto Federal de Pará }\end{array}$ & Pará & & 1 \\
\hline Total & & & 12 \\
\hline
\end{tabular}

A continuación, la Tabla 8 muestra la distribución de los objetos de estudio configurados en instituciones educativas, comunidades rurales y programas gubernamentales que han adoptado la Pedagogía de la Alternancia en sus actividades formativas. Los resultados mostraron que las instituciones educativas $(n=10)$ fueron la mayoría entre los objetos de estudio de la Pedagogía de la Alternancia, seguidas de los programas gubernamentales $(n=7)$, entre ellos PRONAT, PIBID, PROJOVEM, CEDEJOR, PROJOVEM CAMPO, PROCAMPO y PRONERA, así como comunidades rurales $(n=2)$ en las distintas regiones del país. 


\section{Tabla 8}

Otros objetos de estudio que adoptaron la Pedagogía de la Alternancia

\begin{tabular}{|c|c|c|c|}
\hline $\begin{array}{l}\text { Instituciones, comunidades rurales y programas } \\
\text { gubernamentales }\end{array}$ & Estado & Región & Estudios \\
\hline Escuela Municipal Casa Escuela de la Pesca en Outeiro & Pará & \multirow{2}{*}{$\begin{array}{l}\text { Norte } \\
(n=4)\end{array}$} & 2 \\
\hline Centro Educacional de lata en Guajará-Mirim & Rondônia & & 2 \\
\hline Escuela Estadual Rural Taylor-Egídio en Jaguaquara & \multirow[b]{2}{*}{ Bahia } & \multirow{3}{*}{$\begin{array}{l}\text { Noreste } \\
(n=2)\end{array}$} & 2 \\
\hline $\begin{array}{l}\text { PRONAT - Programa de Apoyo al Desarrollo Sostenible de } \\
\text { Territorios Rurales en Salvador }\end{array}$ & & & 1 \\
\hline Escuela Municipal João Joca de Assunção en Timon & Maranhão & & 1 \\
\hline Escuela Comunitaria Agroecológica Piedra Torta en Águia Branca & \multirow{3}{*}{$\begin{array}{l}\text { Espírito } \\
\text { Santo }\end{array}$} & \multirow{6}{*}{$\begin{array}{l}\text { Sureste } \\
(n=8)\end{array}$} & 1 \\
\hline $\begin{array}{l}\text { Escuela Comunitaria Rural Padre Fulgêncio de Menino Jesús, en } \\
\text { Colatina }\end{array}$ & & & 2 \\
\hline Comunidad Rural de la Región Norte del Espírito Santo & & & 2 \\
\hline $\begin{array}{l}\text { PIBID (Programa Institucional de Becas de Iniciación a la Docencia) } \\
\text { Diversidad de la Universidade Federal dos Vales do Jequitinhona e } \\
\text { Mucuri }\end{array}$ & Minas Gerais & & 1 \\
\hline $\begin{array}{l}\text { PROJOVEM/Programa de Formación de Jóvenes Empresarios } \\
\text { Rurales }\end{array}$ & \multirow[t]{2}{*}{ São Paulo } & & 1 \\
\hline Asentamiento Laudenor de Souza en Piratininga & & & 1 \\
\hline CEDEJOR - Centro de Desarrollo del Joven Rural & Paraná & \multirow{5}{*}{$\begin{array}{l}\text { Sur } \\
(n=7)\end{array}$} & 1 \\
\hline $\begin{array}{l}\text { Escuela de Enseñanza Fundamental Construindo o Caminho en } \\
\text { Dionísio Cerqueira }\end{array}$ & \begin{tabular}{|l} 
Santa \\
Catarina \\
\end{tabular} & & 2 \\
\hline Instituto de Educación Josué de Castro en Veranópolis & \multirow{3}{*}{$\begin{array}{l}\text { Rio Grande } \\
\text { do Sul }\end{array}$} & & 2 \\
\hline Proyecto Educación para Pescadores en Rio Grande & & & 1 \\
\hline $\begin{array}{l}\text { Territorios de la Ciudadanía en el Polo de Educación a Distancia } \\
\text { (EaD), de Canoinhas }\end{array}$ & & & 1 \\
\hline Escuela de Teatro Político y Vídeo Popular del Distrito Federal & $\begin{array}{l}\text { Distrito } \\
\text { Federal } \\
\end{array}$ & \multirow{3}{*}{$\begin{array}{l}\text { Centro- } \\
\text { Oeste } \\
(n=2)\end{array}$} & 1 \\
\hline Escuela Municipal Rural Minuano, en Terra Nova do Norte & Mato Grosso & & 1 \\
\hline PROJOVEM CAMPO Saberes de la Tierra, en Cipolândia & $\begin{array}{l}\text { Mato Grosso } \\
\text { do Sul }\end{array}$ & & 1 \\
\hline $\begin{array}{l}\text { PROCAMPO - Programa de Apoyo a la Formación Superior en } \\
\text { Licenciatura en Educación del Campo; PRONERA - Programa } \\
\text { Nacional de Reforma Agraria }\end{array}$ & Brasil & & 2 \\
\hline Total & & & 27 \\
\hline
\end{tabular}

En cuanto a los artículos del tipo "ensayos teóricos" ( $n=60)$, estos fueron publicados entre 1983 y 2019, con una incidencia menor ( $n=6)$ para los publicados entre 1983 y 2005 , abordando los siguientes temas: educación agrícola en Brasil (Argumedo, 1983), Maison Familiales Rurales en Francia (Chartier, 1985; Marois, 2003), Escuelas Familiares Rurales en España (Muñoz- 
Peinado, 2003), Casas Familiares Rurales (Colossi y Estevam, 2003) y Pedagogía de la Alternancia (Azevedo, 2005).

Los demás ensayos teóricos $(n=54)$ fueron publicados entre 2007 y 2019 , como se muestra en el Cuadro 1, elaborados a partir de una muestra intencional de artículos ( $n=13$ ).

\section{Cuadro 1}

Sintesis de estudios teóricos publicados entre 2007 y 2019

\begin{tabular}{|c|c|}
\hline Autores/Año & Objetivo y perspectivas teóricas \\
\hline Nosella (2007) & $\begin{array}{l}\text { Afirma que todo acto pedagógico contiene en sí mismo alguna militancia política } \\
\text { implícita, y la PA efectiva es una opción política progresista, renovadora y revolucionaria, } \\
\text { que busca, además de la plena libertad de opción del hombre del campo, el cambio } \\
\text { profundo de la relación entre campo-ciudad, solidificando una cultura democrática. }\end{array}$ \\
\hline Ribeiro (2008) & $\begin{array}{l}\text { Aborda la educación del campo y rural generada en los movimientos sociales populares, } \\
\text { para plasmar, en las experiencias formativas que articulan trabajo-educación realizadas } \\
\text { por estos movimientos y organizaciones, las contradicciones expresadas en las prácticas } \\
\text { y concepciones de la PA. }\end{array}$ \\
\hline Silva (2009) & $\begin{array}{l}\text { Utiliza representaciones sociales para analizar las relaciones escuela-familia en EFAs y } \\
\text { CFRs, revelando diferentes lógicas en el sentido atribuido a la alternancia. }\end{array}$ \\
\hline Mazzeu (2010) & $\begin{array}{l}\text { Señala los aportes de la PA, desde la perspectiva teórica de Paulo Freire, para repensar la } \\
\text { formación de recolectores de materiales reciclables. }\end{array}$ \\
\hline $\begin{array}{l}\text { Nascimento } \\
(2011)\end{array}$ & $\begin{array}{l}\text { Presenta un análisis epistemológico y crítico basado en la teoría marxista sobre la EC, a } \\
\text { través de un debate teórico y filosófico sobre la categoría "emancipación", discutiendo si } \\
\text { sus prácticas constituyen una fuerza contraria al espíritu del capitalismo. }\end{array}$ \\
\hline $\begin{array}{l}\text { Palaro y Bernartt } \\
\text { (2012) }\end{array}$ & $\begin{array}{l}\text { Se discute la categoría del trabajo como principio educativo y como principio de } \\
\text { alienación en la PA, concluyendo que no puede verse como una praxis revolucionaria, } \\
\text { sino como la adaptación del capital a una clase alejada del mercado capitalista } \\
\text { globalizado. }\end{array}$ \\
\hline $\begin{array}{l}\text { Martins } \\
(2013)\end{array}$ & $\begin{array}{l}\text { Tiene como objetivo dar visibilidad a la Pedagogía de la Tierra, la política y las prácticas } \\
\text { de educación superior vinculadas a una perspectiva emancipadora orientada a las clases } \\
\text { populares. }\end{array}$ \\
\hline $\begin{array}{l}\text { Vergutz y } \\
\text { Cavalcante (2014) }\end{array}$ & $\begin{array}{l}\text { Busca aproximaciones conceptuales a la praxis de la PA para percibir las singularidades } \\
\text { de los aprendizajes fortalecidos en el movimiento alterno potenciado por una } \\
\text { organización pedagógica basada en el diálogo problematizador de Paulo Freire. }\end{array}$ \\
\hline $\begin{array}{l}\text { Belo y Jesus } \\
(2015)\end{array}$ & $\begin{array}{l}\text { Analiza la política de educación rural en Brasil y la adopción de la PA como una práctica } \\
\text { pedagógica que problematiza el reconocimiento de la identidad del conocimiento local. }\end{array}$ \\
\hline $\begin{array}{l}\text { Pinho y } \\
\text { Nascimento } \\
\text { (2016) }\end{array}$ & $\begin{array}{l}\text { Aborda la PA desde la perspectiva del paradigma de la interdisciplinariedad y } \\
\text { transdisciplinariedad, para mostrar que este proceso permite al alumno permanecer en } \\
\text { la escuela en consonancia con el entorno en el que vive, valorando aspectos culturales } \\
\text { de las personas que viven en el campo. }\end{array}$ \\
\hline $\begin{array}{l}\text { Oliveira y Valadão } \\
\text { (2017) }\end{array}$ & $\begin{array}{l}\text { Discute la relevancia de considerar la mediación como foco de análisis de la } \\
\text { heterogeneidad en la Teoría Actor-Red (TAR) en los estudios organizacionales, }\end{array}$ \\
\hline
\end{tabular}




\begin{tabular}{|l|l|}
\hline & $\begin{array}{l}\text { enfrentándola con dos perspectivas paradigmáticas: simplificación y complejidad, } \\
\text { tomando como ejemplo la PA. }\end{array}$ \\
\hline Cambi (2018) & $\begin{array}{l}\text { Analiza la alternancia escuela/trabajo desde la perspectiva de Gramsci como un nuevo } \\
\text { principio educativo para el hombre moderno, vinculado a la lucha de clases, el papel de } \\
\text { la ciencia y la técnica y la propia evolución de la civilización humana. }\end{array}$ \\
\hline $\begin{array}{l}\text { Gomes, Araújo y } \\
\text { Rocha (2019) }\end{array}$ & $\begin{array}{l}\text { Reflexiona sobre las "banderas" fundamentales de los campesinos: la cuestión de la } \\
\text { tierra y la educación rural a la luz de las Constituciones Federales brasileñas. }\end{array}$ \\
\hline
\end{tabular}

El Cuadro 1 muestra los enfoques teóricos adoptados en estos ensayos, tales como: la PA como opción política, progresista, renovadora y revolucionaria, y la PA como articulación entre trabajo y educación. También hay perspectivas teóricas basadas en representaciones sociales, en referencias teóricas Freirianas y Gramscianas, en teorías marxistas, en teoría actor-red, en el paradigma de la interdisciplinariedad y transdisciplinariedad y en la pedagogía de la tierra para abordar la Pedagogía de la Alternancia.

Los artículos de "metanálisis" ( $n=8$ ) se publicaron entre 2008 y 2018. El Cuadro 2, a continuación, presenta un resumen de estos estudios destacando sus principales características.

\section{Cuadro 2}

Principales características de los metanálisis sobre la Pedagogía de la Alternancia

\begin{tabular}{|c|c|c|c|}
\hline Autores/Año & Fuente de datos & Periodo/corpus & Temáticas/Categorías de análisis \\
\hline $\begin{array}{l}\text { Teixeira, Bernartt } \\
\text { y Trindade } \\
\text { (2008) }\end{array}$ & $\begin{array}{l}\text { Banco de Tesis de } \\
\text { la CAPES y otras } \\
\text { fuentes }\end{array}$ & $\begin{array}{l}1969-2006 \\
40 \text { (39TM+7TD) }\end{array}$ & $\begin{array}{l}\text { 1) PA y EC; 2) PA y desarrollo; 3) Implantación de } \\
\text { CEFFAs en Brasil; 4) Relaciones entre CEFFAs y } \\
\text { familias }\end{array}$ \\
\hline $\begin{array}{l}\text { Souza } \\
(2008)\end{array}$ & $\begin{array}{l}\text { Banco de Tesis de } \\
\text { la Capes }\end{array}$ & $\begin{array}{l}1987-2007 \\
170(125 T M \\
+45 T D) \\
\end{array}$ & $\begin{array}{l}\text { 1) EC; 2) MST; 3) Movimientos sociales; 4) } \\
\text { Extensión rural; 5) EJA; 6) PRONERA; 7) formación } \\
\text { de profesores y currículo. }\end{array}$ \\
\hline $\begin{array}{l}\text { Silva y Sahr } \\
(2017)\end{array}$ & BDTD/Ibict & $\begin{array}{l}2007-2014 \\
40 \text { (TD\&TM) }\end{array}$ & $\begin{array}{l}\text { 1) PA y desarrollo regional; 2) Implantación de las } \\
\text { CEFFAs; 3) Relación CEFFAs y familias }\end{array}$ \\
\hline $\begin{array}{l}\text { Nahirne y } \\
\text { Strieder (2017) }\end{array}$ & $\begin{array}{l}\text { Página web de } \\
\text { Periódicos Capes }\end{array}$ & $\begin{array}{l}2008-2015 \\
26 \text { artículos }\end{array}$ & $\begin{array}{l}\text { 1) Origen y trayectoria de la EC; 2) PA como } \\
\text { propuesta metodológica para la organización de } \\
\text { la educación escolar; 3) Plan de estudios de las } \\
\text { escuelas rurales; 4) Políticas públicas y } \\
\text { movimientos sociales de la EC; 5) Lucha de la } \\
\text { población rural por la EC; 6) Graduados }\end{array}$ \\
\hline $\begin{array}{l}\text { Dias y Leonel, } \\
2018\end{array}$ & $\begin{array}{l}\text { ENPEC, SNEF, } \\
\text { EPEF (eventos) }\end{array}$ & $\begin{array}{l}\text { 2002-2016 } \\
\text { nueve artículos }\end{array}$ & $\begin{array}{l}\text { 1) PA y EFA; 2) Estrategias didáctico- } \\
\text { metodológicas para la enseñanza de Física en las } \\
\text { escuelas del campo }\end{array}$ \\
\hline $\begin{array}{l}\text { Ferrari y Ferreira, } \\
2018\end{array}$ & $\begin{array}{l}\text { Banco de Tesis de } \\
\text { la Capes }\end{array}$ & $\begin{array}{l}2007-2013 \\
73(63 T M+10 T D)\end{array}$ & $\begin{array}{l}\text { 1) PA y EC; 2) PA y desarrollo regional; 3) } \\
\text { Implantación de las (EFFAs; 4) Relación CEFFAs y } \\
\text { familias }\end{array}$ \\
\hline
\end{tabular}




\begin{tabular}{|l|l|l|l|}
\hline $\begin{array}{l}\text { Miano y Corro, } \\
2018\end{array}$ & $\begin{array}{l}\text { Bases de datos: } \\
\text { Science Direct; } \\
\text { JSTOR; Springer, } \\
\text { Sage }\end{array}$ & $\begin{array}{l}2011-2017 \\
59 \text { artículos }\end{array}$ & $\begin{array}{l}\text { 1) PA y desarrollo local-rural; 2) instrumentos } \\
\text { específicos de PA; 3) la PA y la formación de un } \\
\text { sujeto crítico; 4) Análisis histórico de los } \\
\text { procesos de apertura de escuelas de alternancia; } \\
\text { 5) PA y educación para el trabajo. }\end{array}$ \\
\hline $\begin{array}{l}\text { Saul, Rodrigues y } \\
\text { Auler (2019) }\end{array}$ & $\begin{array}{l}\text { Banco de Tesis de } \\
\text { la Capes }\end{array}$ & $\begin{array}{l}\text { 2013-2015 } \\
\text { 4 (2TM \& 2TD) }\end{array}$ & $\begin{array}{l}\text { 1) Licenciaturas en Educación Rural y PA; 2) } \\
\text { Formación de profesores; 3) Concepciones y } \\
\text { prácticas. }\end{array}$ \\
\hline
\end{tabular}

TM= Tesis de Magíster; $T D=$ Tesis de Doctorado; $P A=$ Pedagogía de la Alternancia; $E C=$ Educación del Campo.

Se puede observar, en el Cuadro 2, que la mayoría de los metanálisis ( $n=5)$ tuvieron como objeto de estudio tesis de magíster y de doctorado, seleccionadas en el Banco de Tesis de la CAPES y en la Biblioteca Digital Brasileña de Tesis del Instituto Brasileño de Información en Ciencia y Tecnología (BDTD/Ibict). Cabe señalar que el estudio de Teixeira et al. (2008) también recogió datos en sitios web de programas de posgrado en educación en Brasil, en Internet y en listas de referencias bibliográficas de tesis de magíster y de doctorado con texto completo analizado. El número total de artículos analizados en estos metanálisis $(n=333)$ incluyó tesis de magíster $(n=$ $229)$, tesis de doctorado $(n=64)$ y un corpus $(n=40)$ que no discriminó la distribución entre trabajos de maestría y de doctorado. Además, los metanálisis $(n=3)$ también analizaron 85 artículos de revistas y nueve trabajos presentados en eventos científicos en el área de Educación Física y Ciencias, incluyendo el Encuentro Nacional de Investigación en Educación en Ciencias (ENPEC), Simposio Nacional de la Enseñanza de Física (SNEF) y Enseñanza de la Física (EPEF).

Teniendo en cuenta el marco temporal de todos los metanálisis $(n=8)$, estos cubrieron un período extenso entre 1969 y 2017. En cuanto a los temas y categorías de análisis de estos estudios, las relaciones entre PA y Educación del Campo, PA y MST, licenciaturas en PA, formación docente, el currículo de la PA y de la EC, entre otros.

La lectura completa de los metanálisis de la producción científica sobre Pedagogía de la Alternancia permitió identificar los principales hallazgos de estos estudios (Cuadro 3).

\section{Cuadro 3}

Principales hallazgos y resultados de los estudios de metanálisis

\begin{tabular}{|l|l|}
\hline Autores/Año & Principales hallazgos y resultados \\
\hline \multirow{3}{*}{$\begin{array}{l}\text { Teixeira, } \\
\text { Bernartt y } \\
\text { Trindade } \\
(2008)\end{array}$} & $\begin{array}{l}\text { La temática de la PA prevalece desde la perspectiva del desarrollo y fortalecimiento de la } \\
\text { amplementación de los CEFFAs está incluido en estudios de caso realizados en el país. }\end{array}$ \\
& $\begin{array}{l}\text { Otros estudios abordan la dinámica de interacción entre familias y CEFFA como elemento } \\
\text { fundamental. Sugerimos estudios más profundos sobre la dinámica de la relación } \\
\text { familia-CEFFA, los fundamentos teórico-metodológicos de la PA y las relaciones entre los } \\
\text { CEFFAs y el Estado. }\end{array}$ \\
\hline
\end{tabular}




\begin{tabular}{|c|c|}
\hline Souza (2008) & $\begin{array}{l}\text { Son más recurrentes temas sobre aspectos de la educación escolar, la dimensión } \\
\text { sociopolítica de los movimientos sociales y factores culturales relacionados con la } \\
\text { sexualidad, el género, la juventud y la religión. Las retrospectivas históricas del MST son } \\
\text { similares y repetitivas, lo que sugiere la valoración de síntesis sobre la lucha por la tierra } \\
\text { en Brasil. }\end{array}$ \\
\hline $\begin{array}{l}\text { Ferrari y } \\
\text { Ferreira (2016) }\end{array}$ & $\begin{array}{l}\text { Los temas de mayor interés son la relación entre PA y EC, y el desarrollo del medio } \\
\text { ambiente. Muestra la creciente adopción de la PA en contextos educativos y escolares en } \\
\text { los que no suele ser tradicional, como Institutos Federales y Universidades y, también, su } \\
\text { articulación con la EJA. }\end{array}$ \\
\hline $\begin{array}{l}\text { Silva y Sahr } \\
\text { (2017) }\end{array}$ & $\begin{array}{l}\text { La creación de programas de posgrado en instituciones regionales puede haber tenido un } \\
\text { impacto en la mayor dispersión de la producción científica, aunque aún existe un } \\
\text { desajuste entre el número y distribución de los CEFFAS en relación con la producción de } \\
\text { discusiones académicas. }\end{array}$ \\
\hline $\begin{array}{l}\text { Nahirne y } \\
\text { Strieder (2017) }\end{array}$ & $\begin{array}{l}\text { Destaca los importantes logros en el ámbito de la legislación brasileña en el campo de la } \\
\text { Educación Rural, mostrando dificultades para estructurar un perfil educativo compatible } \\
\text { con dicha legislación y las demandas educativas de la población insertada en este } \\
\text { contexto. }\end{array}$ \\
\hline $\begin{array}{l}\text { Dias y Leonel } \\
\text { (2018) }\end{array}$ & $\begin{array}{l}\text { El enfoque CTS y la Etnofísica fueron identificados como la estrategia metodológica para } \\
\text { abordar los temas, siendo esta última la más utilizada en el contexto de las EFAs, ya que } \\
\text { posibilita la relación entre la Física que se estudia en el aula y la realidad de los } \\
\text { estudiantes. }\end{array}$ \\
\hline $\begin{array}{l}\text { Miano y } \\
\text { Corro (2018) }\end{array}$ & $\begin{array}{l}\text { Los resultados mostraron que Brasil concentra el mayor número de investigaciones en } \\
\text { PA, seguido en menor medida por Argentina, con una reciente tendencia productiva en } \\
\text { Perú. Hay una línea temática específica en Brasil relacionada con la alternancia y la } \\
\text { formación de un sujeto crítico enraizado en las áreas rurales. Los resultados mostraron } \\
\text { que el campo de investigación de la PA tiene perspectivas muy diferentes e incluso } \\
\text { contrastantes, entre las que se pueden identificar los polos "pedagogía de la alternancia y } \\
\text { emancipación" y "pedagogía de la alternancia y sujeción". }\end{array}$ \\
\hline $\begin{array}{l}\text { Saul, Rodrigues } \\
\text { y Auler (2019) }\end{array}$ & $\begin{array}{l}\text { Las producciones académicas analizaron el diálogo con las referencias sobre PA, sus } \\
\text { supuestos y análisis prácticos. La PA se considera fundamental y necesaria para la EC de } \\
\text { nivel básico y superior. }\end{array}$ \\
\hline
\end{tabular}

Cabe señalar que los principales hallazgos de estos metanálisis se refieren a la producción científica sobre CEFFAs $(n=2)$, Educación del Campo $(n=2)$, MST $(n=1)$, Educación de Jóvenes y Adultos (EJA) $(n=1)$ y la perspectiva del movimiento CTS, es decir, Ciencia, Tecnología y Sociedad ( $n=1$ ). En la Tabla 3, aún es posible verificar que solo un metanálisis (Miano y Corro, 2018) asumió la perspectiva de un estudio comparativo cotejando la producción científica de ocho países de América Latina (Argentina, Brasil, Colombia y Perú), Centroamérica (Guatemala) y Europa (España, Francia e Italia). Estudios con estas características avanzan en el análisis del conocimiento producido sobre la PA porque permiten identificar las articulaciones teóricas y los debates que están implícitos en este campo. 


\section{Conclusiones}

Durante cincuenta años, desde la introducción de la PA en Brasil, ha habido una enorme producción científica sobre este tema, como lo demuestra el corpus de investigación representado por artículos ( $n=251$ ) publicados entre 1981 y 2019 en revistas científicas brasileñas $(n=120)$ y en el extranjero $(n=37)$. Esta producción científica ha crecido significativamente desde 2007.

En cuanto a la autoría, la mayoría de los artículos ( $n=174)$ son en coautoría, predominando el género femenino $(n=240)$ entre los autores $(n=408)$. Los estudios de caso $(n=$ 183) predominaron entre los tipos de artículos y se realizaron en municipios $(n=208)$ de las regiones sur y sureste de Brasil. Los estudios de caso eligieron EFAs ( $n=104)$ y CFRs $(n=52)$ como objeto de estudio, seguidos de cursos de licenciatura $(n=45)$, instituciones de enseñanza, comunidades, programas gubernamentales $(n=27)$ y cursos técnicos de perfeccionamiento y especialización ( $n=12$ ) que adoptan la Pedagogía de la Alternancia en su ámbito. También, se realizaron ensayos teóricos $(n=60)$ que adoptaron diferentes perspectivas teóricas para analizar la PA, así como metanálisis $(n=8)$ que analizaron la producción científica consistente en tesis de doctorado, tesis de magíster y artículos científicos publicados en Brasil y en el exterior, abarcando el período comprendido entre 1969 y 2017.

Investigaciones futuras sobre la producción científica de la Pedagogía de la Alternancia podrían profundizar los hallazgos aquí expuestos mediante la realización de un estudio de citas de los artículos, ya que esto permitiría indagar en mayor profundidad los referentes teóricos que constituyen la base teórica y metodológica de los estudios analizados. Además, futuros estudios podrían utilizar otras bases de datos (por ejemplo, Dimensions, Google Scholar, entre otras) para ampliar el corpus analizado y comparar los resultados obtenidos.

Finalmente, los análisis bibliométricos y de contenido permitieron identificar las perspectivas longitudinales y estructurales de la producción científica en Pedagogía de la Alternancia, tomando en cuenta el perfil de los/as autores/as y de las autorías, las revistas que difunden estos trabajos, las investigaciones desarrolladas en estos estudios, así como su vínculo con varios contextos disciplinarios e interdisciplinarios. En este sentido, la identificación del estándar de desarrollo de esta base de conocimiento reveló algunos puntos de saturación, por ejemplo, en relación con los objetos de estudio y las fuentes de datos de los metanálisis. Estos resultados pueden contribuir a nuevas lecturas sobre la Pedagogía de la Alternancia y motivar futuras inversiones intelectuales tanto para los/as autores/as que ya pertenecen a este campo de estudio como para aquellos/as que pretenden dedicarse a investigaciones sobre este tema. 


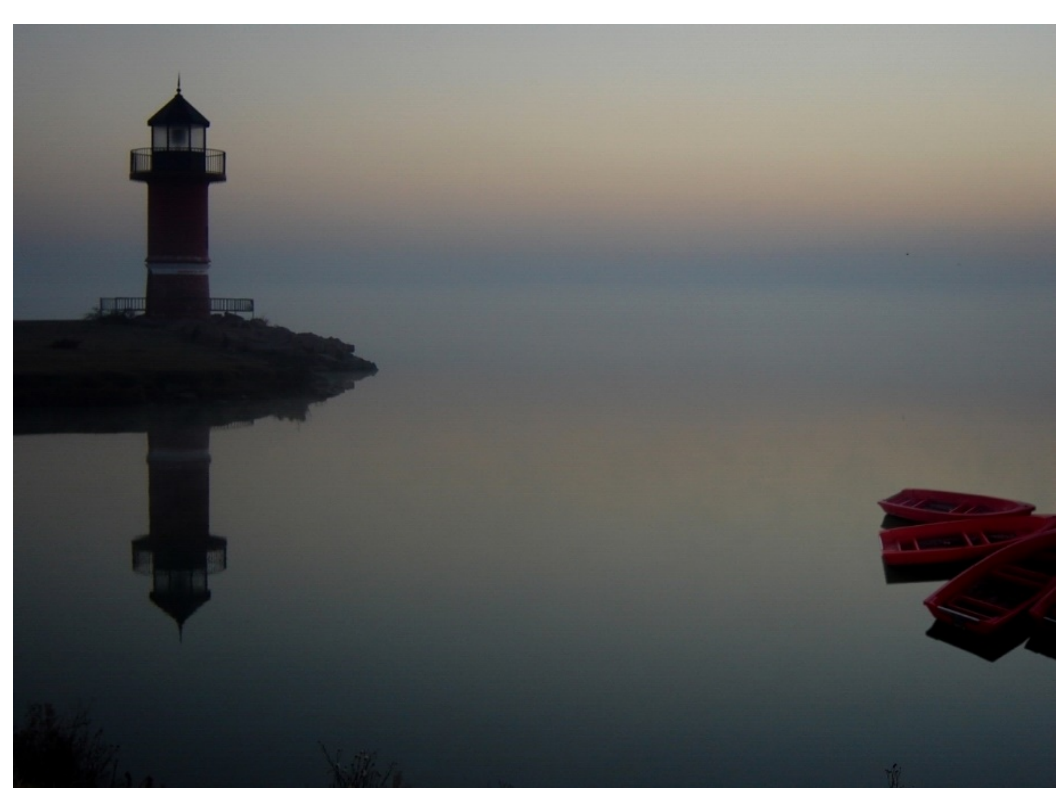

\section{Laguna y niebla, fotografía. Alejandro Urioste}

\section{Notas}

(1) Agradecemos a Paolo Nosella por la cesión de copias de esos artículos.

\section{Bibliografía}

American Psychiatric Association (APA). (2012). Manual de publicação da APA. Penso.

Argumedo, M. A. (1983). O ensino agrícola no Brasil: uma estratégia educacional. Educação em Debate, $(4 / 5) 2 / 1,55-90$.

Azevedo, A. J. (2005). Sobre a pedagogia da alternância. Revista Científica Eletrônica de Pedagogia, 6(3), 1-10. Belo, M. S. S. P. y Jesus, L. F. (2015). A construção da identidade pedagógica na educação do campo. Olhar de Professor, (18)2, 221-235

Bicalho, R. (2013). Educação do campo e pedagogia da alternância. Educere et Educare, (8)5, 45-58.

Cambi, F. (2018). Note sulla scuola attuale: sull'alternanza scuola-lavoro. Studi della Formazione, (27), $233-235$. Chartier, D. (1985). La spécificité des Maisons Familiares Rurales d'éducation et d'orientation. Revue Française de Pédagogie, (73), 23-30.

Colossi, N. y Estevam, D. O. (2003). Casas familiares rurais: uma alternativa para a formação de jovens agricultores. Revista de Administração, (2)3, 15-42.

Coordenação de Aperfeiçoamento de Pessoal de Nível Superior (CAPES). (2020). Aprimoramento do processo de avaliação da pós-graduação. 2019. Recuperado de:

http://uploads.capes.gov.br/files/Oficio_1015556.pdf Acceso el 10 de diciembre de 2020.

Dias, F. F. y Leonel, A. A. (2018). Escolas de campo: um olhar sobre a legislação e práticas implementadas no Ensino de Física. Ensaio: Pesquisa em Educação em Ciências, (20)2874, 1-22.

Ferrari, G. M. y Ferreira, O. S. (2016). Pedagogia da alternância nas publicações acadêmicas no Brasil (20072013). Revista Brasileira de Educação do Campo, ( )2, 495-523.

García-Marirrodriga y Calvó, P. P. (2010). Formação em alternância e desenvolvimento local: o movimento educativo dos CEFFAS no mundo. O Lutador. 
Gioanordoli, R. L. (1980). Nova perspectiva para a educação rurał. pedagogia da alternância. 1980. Dissertação (Mestrado em Educação). PUC-R).

Gimonet, J. C. (2007). Praticar e compreender a pedagogia da alternância dos CEFFAs. Vozes.

Gomes, E. M., Araújo, A. F. y Rocha, M. I. A. (2019). Legislação sobre a posse da terra e educação no campo no Brasil. Revista Brasileira de Educação do Campo, (4)e6406, 2019.

Gonçalves, T. L. y Hayashi, M. C. P. I. (2016). Educação do campo: contribuições para o estado da arte da produção científica (2007-2015). Revista da FAEEBA, (25)46, 207-225.

Gonçalves, T. L. y Hayashi, M. C. P. I. (2014). Estudo bibliométrico sobre educação do campo para jovens e adultos deficientes. Série-Estudos (UCDB), (38), 129-149.

Hayashi, M. C. P. I y Gonçalves, T. L. (2016). Perfil bibliométrico dos grupos de pesquisa em Educação do Campo (2000-2016). Revista Brasileira de Educação do Campo, ( \1, 4-25.

Hayashi, M. C. P. I. y Gonçalves, T. G. G. L. (2018). Estudo bibliométrico dos balanços da produção científica em Educacão Especial na Revista Brasileira de Educação Especial (1999-2017). Revista Brasileira de Educação Especial, (24), 135-152.

Leal, E. J. M. (2011). Produção acadêmico-científica: a pesquisa e o ensaio. Universidade do Vale do Itajaí. Lopes, A. C. y Costa, H. H. C. (2012). A produção bibliográfica na área de educação. Revista Brasileira de Educação, (17)51, 717-730, 2012.

Marois, T. (2020). La pédagogie de l'alternance en maisons familiales rurale. Recherches \& Éducation, 4(1). Recuperado de: https://journals.openedition.org/rechercheseducations/187 Acceso el 10 de diciembre de 2020 .

Maroldi, A. M., Lima, L. F. M., Hayashi, C. R. M. y Hayashi, M. C. P. I. (2017). Panorama bibliométrico das teses e dissertações sobre educação indígena. Revista Brasileira de Educação do Campo, (2)2, 677-707.

Maroldi, A. M., Lima, L. F. M. y Hayashi. (2018). Análise de citações presentes em teses e dissertações em educação indígena. Informação \& Informação, (23)3, 177-201.

Martins, F. J. (2013). Pedagogía de la tierra: los sujetos del campo y la enseñanza. Revista NuestrAmérica, ( $) 2$, 67-85.

Mazzeu, F. J. C. (2010). Educação e economia solidária: contribuições da pedagogia da alternância para a formação dos catadores de materiais recicláveis. Revista da FAEEBA, (19)34, 49-62.

Miano, M. A. y Corro, E. S. L. (2018). Temáticas, geografias y debates en el campo de la pedagogia de la alternancia. Revista de Investigación Educativa, (27), 60-89.

Michel, M. H. (2015). Metodologia e pesquisa científica em ciências sociais: um guia prático para acompanhamento da disciplina e elaboração de trabalhos monográficos. Atlas.

Muñoz-Peinado, J. (2003). Los colégios familiares rurales. Revista Interuniversitária de Formación del Profesorado, $(17) 3,75-89$.

Nascimento, C. G. (2011). Educação do campo na encruzilhada entre emancipação versus reino do capital: uma leitura filosófica. Revista NERA, (14)18, 106-124.

Nahirne, A. P. y Strieder, D. M. (2017). Metaestudo da produção científica sobre a escola e a educação do campo. Educere et Educare, (12)24, 1-15.

Nosella, P. (1970). Chance aos agricultores: o Movimento de Educação Promocional do Espírito Santo.

Cadernos do CEAS, (8), 1-12.

Nosella, P. (1977). Uma nova educação para o meio rural: sistematização e problematização da experiência educacional das escolas da Família Agrícola do Movimento de Educação Promocional do Espírito Santo. Dissertação (Mestrado em Educação). São Paulo: PUC-SP, 1977. 
Estudio bibliométrico sobre la Pedagogía de la Alternancia en artículos científicos (1981-2019). I

María Cristina Piumbato Innocentini Hayashi; Alexandre Masson Maroldi; Taísa Grasiela Gomes Liduenha Gonçalves; Carlos Roberto

Massao Hayashi.

Nosella, P. (1978). Uma nova educação para o meio rural. Revista de Cultura UFES, ( 1 1, 81-100.

Nosella, P. (2007). Militância e profissionalismo na educação do homem do campo. Revista da Formação por Alternância, (2), 5-17.

Nosella, P. (2012). Educação do campo: origens da pedagogia da alternância no Brasil. UFES.

Oliveira, V. M. y Valadão, J. A. D. (2017). Entre a simplificação e a complexidade, a heterogeneidade: Teoria Ator-Rede e uma nova epistemologia para os estudos organizacionais. Cadernos EBAPE.BR, (15)4, 877-899.

Palaro, R. y Bernartt, M. L. (2012). 0 trabalho como princípio educativo e como princípio de alienação: possibilidades e limites da pedagogia da alternância. Revista Histedbr Online, (12)46, 293-308.

Pessotti, A. L. (1978). Escola da Família Agrícola: uma alternativa para o ensino rural. 1978. Dissertação (Mestrado em Educação). Fundação Getúlio Vargas: Rio de Janeiro.

Pessotti, A. L. (1981). Escola-família: a pedagogia da alternância no meio rural. Fórum Educacional, (5)2, p. 3959.

Pinho, M. J. y Nascimento, E. P. (2016). Pedagogia da alternância e a prática inter e transdisciplinar em escolas do campo. Revista Eventos Pedagógicos, ( $) 3,1484-1505$.

Pizzani, L., Silva, R. C., Bello, S. F. y Hayashi, M. C. P. I. (2012). A arte da pesquisa bibliográfica na busca do conhecimento. Revista Digital de Biblioteconomia e Ciência da Informação, (10)2, 53-66.

Ribeiro, M. (2010). Movimento camponês, trabalho e educação - liberdade, autonomia, emancipação: princípios/fins da formação humana. Expressão Popular.

Ribeiro, M. (2008). (2008). Pedagogia da alternância na educação rural/do campo: projetos em disputa. Educação e Pesquisa, (34)1, 27-45.

Saul, T. S. y Rodrigues, R. A. y Auler, N. M. F. (2019). A pedagogia da alternância nas licenciaturas em educação do campo: olhar sobre as produções acadêmicas. Revista Brasileira de Educação do Campo, (4)e5541.

Silva, L. H. (2009). Centros familiares de formação por alternância: avanços e perspectivas na construção da educação do campo. Cadernos de Pesquisa: Pensamento Educacional, (4)8, 270-290.

Silva, M. R. y Hayashi, C. R. M. e Hayashi, M. C. P. I. (2011). Análise bibliométrica e cientométrica: desafios para especialistas que atuam no campo. InCID: Revista de Ciência da Informação e Documentação, (2)1, 110-129. Schiebinger, L. (1999). Has feminism changed science? Harvard University Press.

Silva, W. y Sahr, C. L. L. (2017). Os centros educativos familiares de formação em alternância nas reflexões sobre o desenvolvimento: o estado da arte da produção acadêmica brasileira. Ceosul, (32)64, 193-216.

Soares, G. A. D., Souza, C. P. R. y Moura, T. W. (2010). Colaboração na produção científica na Ciência Política e na Sociologia brasileiras. Sociedade e Estado, (25)3, 525-538.

Souza, M. A. (2008). Educação do campo: políticas, práticas pedagógicas e produção científica. Educação \& Sociedade, (29)105, 1089-1111.

Teixeira, E. S. y Bernartt, M. L. e Trindade, G. A. (2008). Estudos sobre a pedagogia da alternância no Brasil: revisão de literatura e perspectivas para a pesquisa. Educação e Pesquisa, (34)2, 227-242.

Vergutz, C. L. B. y Cavalcante, L. O. H. (2014). As aprendizagens na pedagogia da alternância e na educação do campo. Reflexão \& Ação, (22)2, 371-390.

Yin, R. K. (2015). Estudo de caso: planejamento e métodos. Bookman. 